ARTICLE

\title{
The T300A Crohn's disease risk polymorphism impairs function of the WD40 domain of ATG16L1
}

\author{
Emilio Boada-Romero', Inmaculada Serramito-Gómez', María P. Sacristán', David L. Boone², Ramnik J. Xavier ${ }^{3,4}$ \\ $\&$ Felipe X. Pimentel-Muiños ${ }^{1}$
}

A coding polymorphism of human ATG16L1 (rs2241880; T300A) increases the risk of Crohn's disease and it has been shown to enhance susceptibility of ATG16L1 to caspase cleavage. Here we show that T300A also alters the ability of the C-terminal WD40-repeat domain of ATG16L1 to interact with an amino acid motif that recognizes this region. Such alteration impairs the unconventional autophagic activity of TMEM59, a transmembrane protein that contains the WD40 domain-binding motif, and disrupts its normal intracellular trafficking and its ability to engage ATG16L1 in response to bacterial infection. TMEM59-induced autophagy is blunted in cells expressing the fragments generated by caspase processing of the ATG16L1-T300A risk allele, whereas canonical autophagy remains unaffected. These results suggest that the T300A polymorphism alters the function of motif-containing molecules that engage ATG16L1 through the WD40 domain, either by influencing this interaction under non-stressful conditions or by inhibiting their downstream autophagic signalling after caspase-mediated cleavage.

\footnotetext{
${ }^{1}$ Instituto de Biología Molecular y Celular del Cáncer (CSIC-Universidad de Salamanca), Centro de Investigación del Cáncer, Campus Miguel de Unamuno, Universidad de Salamanca, Salamanca 37007, Spain. ${ }^{2}$ Departments of Microbiology and Immunology, Indiana University School of Medicine-South Bend, South Bend, Indiana 46617, USA. ${ }^{3}$ Center for Computational and Integrative Biology, Massachusetts General Hospital, Harvard Medical School, Boston, Massachusetts 02114, USA. ${ }^{4}$ Broad Institute of MIT and Harvard, Cambridge, Massachusetts 02142, USA. Correspondence and requests for materials should be addressed to F.X.P. (email: fxp@usal.es).
} 
C rohn's disease (CD) is a complex genetic and multifactorial condition that involves inflammation of discontinuous areas of the small intestine and colon ${ }^{1-3}$. The pathology is initially episodic, but usually evolves to chronic and refractory states, causing severe complications that may require resective surgery 4,5 . The underlying molecular causes of $\mathrm{CD}$ have remained elusive.

A number of genetic association studies have contributed to the identification of molecules and signalling pathways involved in the onset of this condition ${ }^{6-10}$. Several coding risk polymorphisms identified in such studies correspond to molecules that mediate innate immunity and autophagy, suggesting a role for these processes in the disease ${ }^{3,4,11}$. Autophagy is an intracellular degradation pathway that helps maintain the metabolic homeostasis of the cell ${ }^{12-14}$. A selective form of autophagy, termed xenophagy, plays an important role in innate immunity by promoting the elimination of invading microorganisms ${ }^{15,16}$. Thus, different mechanisms mediate sequestration of infectious agents into autophagic vesicles that eventually fuse with lysosomes for degradation of their contents ${ }^{17-20}$. CD risk mutations involving the innate immune receptor NOD2 lead to an inability to recognize intracellular microorganisms, defective downstream xenophagy and poor defence against infection ${ }^{21}$. In addition, a risk polymorphic form of the critical autophagic effector ATG16L1 (T300A) impairs the autophagic clearance of intracellular pathogens ${ }^{3,4}$. The current paradigm is that dysfunctional xenophagy and altered innate immunity caused by the defective alleles of these two molecules prevents proper control of the commensal intestinal flora and favours the onset of inflammation and CD (refs 4,22,23).

ATG16L1 mediates autophagy by assembling the molecular complex that lipidates LC3 (ref. 24), the main marker of autophagic vesicles $^{25}$. In this complex, the dimer ATG5-ATG12 holds the lipidating activity ${ }^{26}$, whereas ATG16L1 defines the site where LC3 will be lipidated ${ }^{24}$. ATG16L1 includes a C-terminal domain formed by seven WD40-type repetitions (the WD domain, WDD; residues $320-607)^{27}$, the function of which has remained unclear. This region is absent in yeast Atg16, indicating that it is not necessary for the basic functions of autophagy in unicellular organisms ${ }^{27}$. In fact, it appears to be irrelevant for basal or starvation-induced autophagy in mammalian cells, as ATG16L1-deficient cells fully recover these canonical autophagic activities when restored with a version of ATG16L1 that lacks the WD40 repeats ${ }^{28-31}$. Such observation is in line with the fact that most of the critical autophagic effectors that bind ATG16L1 recognize regions other than the $\mathrm{WDD}$, like, for example, ATG5 (which recognizes residues 1-79 of ATG16L1) ${ }^{27}$, FIP200 (residues 230-250 and 288-300 (ref. 28) or 229-242 (ref. 29), WIPI2 (residues 207-230) ${ }^{32}$ and Rab33 (residues 80-265) 33. $^{33}$. Therefore, the function of the WD40 repeats is likely unrelated to canonical autophagy. Instead, it might be related to the various alternative activities recently linked to ATG16L1 through the study of ATG16L1-deficient experimental systems, like inflammatory control ${ }^{34,35}$, trafficking of secretory vesicles in Paneth cells ${ }^{36-38}$ or some forms of xenophagy ${ }^{36,39,40}$.

Recent work from our laboratory showed that the WDD is specifically recognized by a novel 19 -amino acid element found in the intracellular domain of the transmembrane protein TMEM59 (residues 263-281) as well as other molecules ${ }^{41}$. TMEM59 is a glycosylated, widely expressed, type-I transmembrane protein that has been involved in regulating the complex glycosylation of the amyloid precursor protein ${ }^{42}$. We previously reported that TMEM59 is able to engage ATG16L1 to induce an unconventional autophagic process that causes LC3 labelling of the same single-membrane endosomes where TMEM59 is located, thus promoting a more efficient lysosomal targeting of these vesicles ${ }^{41}$. This activity requires four critical positions within the active element (Y268, E272, Y277 and L280) that define a novel motif able to bind the WDD (ref. 41). Such unconventional autophagic event participates in the LC3 labelling of single-membrane phagosomes containing Staphylococcus aureus, suggesting a role in the innate cellular response against invading microorganisms ${ }^{41}$. The WDD of ATG16L1 has also been shown to recognize ubiquitin ${ }^{43}$, an ability that might be common to functionally diverse WD40 domain folds ${ }^{44}$.

How the T300A mutation alters the biology of ATG16L1 has remained unclear. Existing evidence argues that it does not impair, or has relatively minor effects, on basal and starvationinduced autophagy ${ }^{30,39,45}$. Instead, it appears to derail some of the alternative functions carried out by ATG16L1, such as maintenance of the secretory compartment in intestinal Paneth and goblet cells ${ }^{38,46}$, inflammation control ${ }^{34}$ and xenophagy $39,45-49$. Recently, it has been shown that T300A increases susceptibility of ATG16L1 to caspase-3 cleavage in a neighbouring consensus site, leading to decreased levels of full-length ATG16L1 in the context of a stressful situation and, consequently, dysfunctional autophagy and xenophagy ${ }^{46,47}$.

Here we show that, under non-stressful conditions, unprocessed ATG16L1-T300A (hereafter ATG16L1-A300) has an intrinsic reduced ability to bind certain versions of the ATG16L1-binding motif that recognizes the WDD, an alteration that results in defective downstream autophagy, slow intracellular trafficking of motif-containing transmembrane molecules and reduced engagement of ATG16L1 in response to $S$. aureus infection.

\section{Results}

ATG16L1 WDD is required for TMEM59-induced autophagy. To evaluate the functional consequences of caspase-3-mediated processing of ATG16L1 on the activity of molecules that include a WDD-binding motif (for example, TMEM59), we reconstituted ATG16L1 $1^{-1-}$ HCT116 cells ${ }^{45}$ with either intact ATG16L1, the $\mathrm{N}$-terminal or C-terminal portions that result from caspase-3 processing, or both portions simultaneously. We then subjected the resulting cellular strains to TMEM59-induced autophagy or autophagy promoted by conventional stimuli. Interestingly, the ability of TMEM59 to induce lipidation of co-transfected HALC3 was blocked by uncoupling of the $\mathrm{N}$ - and C-terminal domains of ATG16L1 (Fig. 1a). However, clearance of p62-HA remained unaltered in the same conditions (Supplementary Fig. 1a), suggesting that TMEM59-induced autophagy and the basal autophagic flux have different ATG16L1 domain requirements. The autophagic activity of TMEM59 in this setting was restricted to the functional motif that we previously identified in its intracellular domain, since it was retained by a deleted version of the protein exclusively harbouring the 19amino acid minimal active peptide (TMEM59- $\Delta 282)^{41}$ and blocked by mutation of the four critical residues to alanine (4M version $^{41}$; Fig. 1b). Inhibition of TMEM59-induced autophagy by split ATG16L1 was also detected by measuring the translocation of co-transfected green fluorescent protein (GFP)-LC3 (Fig. 1c) or endogenous LC3 (Supplementary Fig. 1b) to a punctated pattern. Notably, basal and rapamycin-induced autophagy proceeded normally provided that the N-terminal portion of ATG16L1 was present, regardless of whether or not it was physically connected to the C-terminal region (Fig. 1d-f). This is consistent with previous reports showing that the $\mathrm{N}$-terminal domain of ATG16L1 suffices to sustain basal autophagy in mammalian cells ${ }^{28-31}$. Taken together, these results argue that ATG16L1 includes two functional modules: an N-terminal effector region 
a

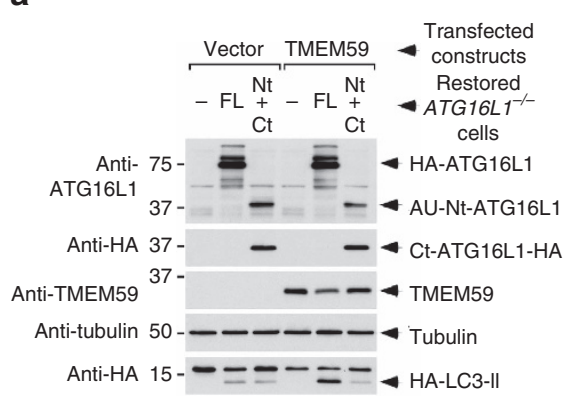

C

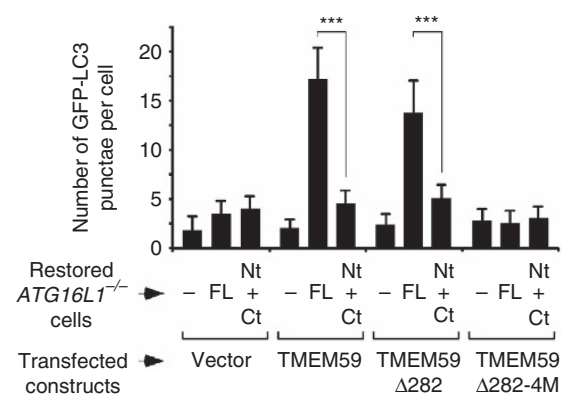

e

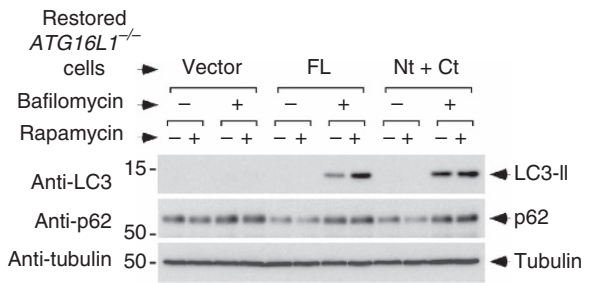

b

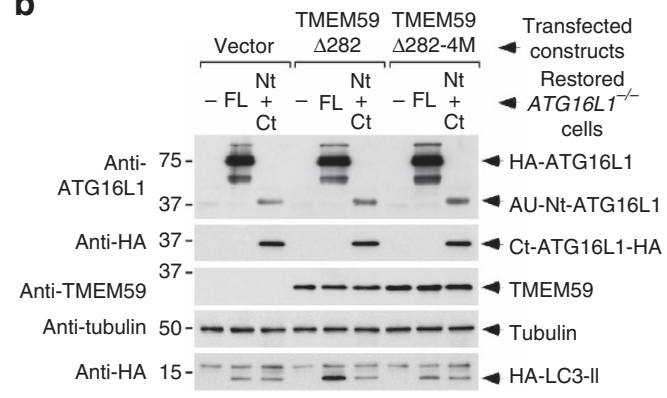

d
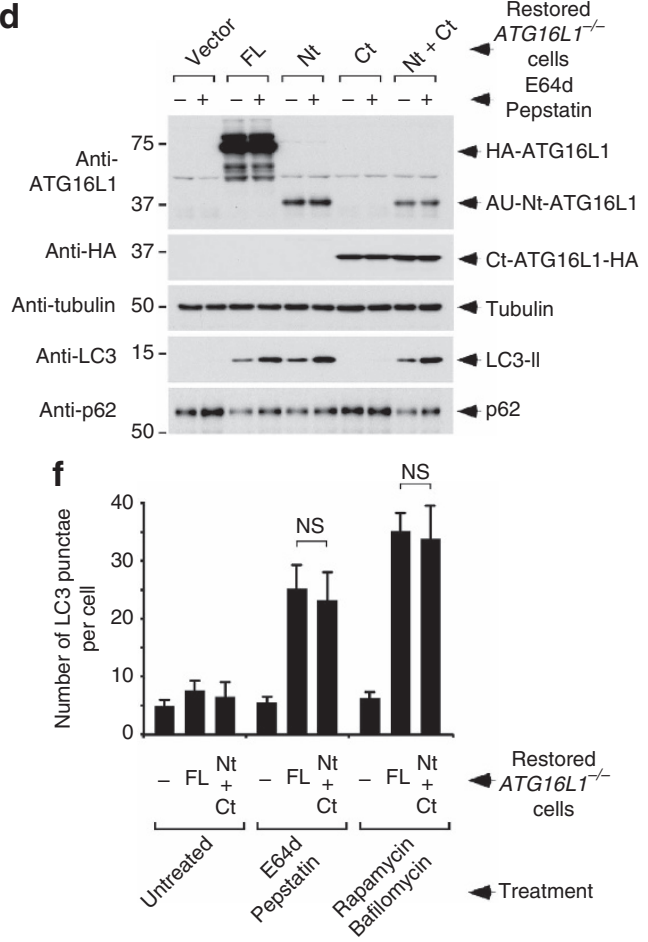

Figure 1 | Impaired TMEM59-induced autophagy in the presence of ATG16L1 fragments resulting from caspase-3-mediated cleavage.

$(\mathbf{a}, \mathbf{b})$ Immunoblot analysis of HA-LC3 lipidation induced by TMEM59 overexpression for $36 \mathrm{~h}$ in ATG16L1-/- HCT116 cells restored with full-length HA-ATG16L1 (FL), both ATG16L1 fragments (Nt: 1-299; Ct: 300-607) or irrelevant vector ( - ). TMEM59- $\Delta 282$ is the largest C-terminal deletion that retains the autophagic potential of the molecule. $4 \mathrm{M}$ designates a mutated form of TMEM59 where the four residues that are essential for its autophagic activity are mutated to alanine. (c) Quantification of GFP-LC3 punctae per transfected cell induced by TMEM59 overexpression for $36 \mathrm{~h}$ in the same cell lines as in $\mathbf{a}$ and $\mathbf{b}$. Shown are mean values \pm s.d. ( $n=50$ cells, ${ }^{\star \star \star} P<0.001$ Student's $t$-test). (d,e) Immunoblot analysis of endogenous LC3 lipidation and p62 expression levels induced in the indicated restored ATG16L1-/- HCT116 cells by treatment with E64d/Pepstatin $\left(10 \mu \mathrm{g} \mathrm{ml} \mathrm{I}^{-1}\right.$ each, $\left.8 \mathrm{~h}\right)$ or bafilomycin $(50 \mathrm{nM}, 8 \mathrm{~h}) \pm$ rapamycin $(2 \mu \mathrm{g} \mathrm{ml}-1,8 \mathrm{~h})$. (f) Quantification of endogenous LC3 punctae per cell induced by the indicated treatments (treatment conditions were as in $\mathbf{d}$ and $\mathbf{e}$; NS, not significant, $P>0.05$ Student's $t$-test). All results shown in this figure are representative of at least two repetitions.

that mediates all autophagic activities of the protein, and a C-terminal WD40 domain that acts as a docking site for upstream inducers of unconventional autophagy, such as TMEM59. In addition, these data suggest that caspase-cleaved ATG16L1 may preferentially impair the autophagic activity of molecules that engage ATG16L1 through the WDD.

ATG16L1-A300 impairs TMEM59-induced autophagy. Recent reports have shown that enhanced processing of ATG16L1-A300 by caspase 3 occurs in response to stressful inducers of autophagy, such as bacterial infection or metabolic stress ${ }^{46,47}$. However, given that position 300 localizes in the vicinity of the WD40 repeats (residues 320-607), we wondered whether the A300 risk polymorphism might influence the unconventional autophagic activities mediated by the WDD under homeostatic conditions with minimal caspase 3 activity. Using ATG16L1 ${ }^{-/-}$
HCT116 cells restored with T300 or A300 forms of ATG16L1 (Supplementary Fig. 2a), we found that TMEM59-induced autophagy was impaired in cells expressing the A300 allele, both at the level of LC3 translocation to a vesiculated pattern (Fig. 2a; Supplementary Fig. 2b) and, less prominently, by measuring LC3 lipidation (Fig. 2b). Again, the levels of co-transfected p62-HA remained unchanged (Supplementary Fig. 2c), suggesting that the autophagic activity of TMEM59 that becomes derailed by ATG16L1-A300 is different from the basal autophagic flux. Consistently, no alterations in basal or rapamycin-induced autophagy were detected in cells harbouring the risk allele (Supplementary Fig. 2d-f). Importantly, the decreased autophagic activity of TMEM59 caused by ATG16L1A300 occurred even in the presence of a pan-caspase inhibitor (Fig. 2c) that blocks caspase-3 activation and ATG16L1 cleavage induced by tumour necrosis factor (TNF; Supplementary Fig. 3a). In addition, cells expressing a mutated version of ATG16L1-A300 
a

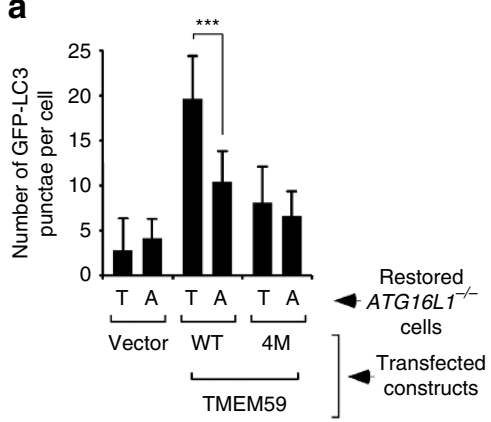

C

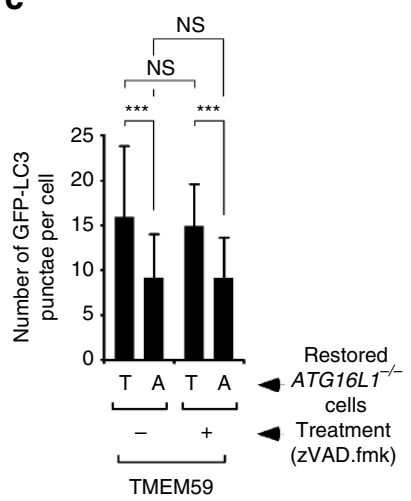

b

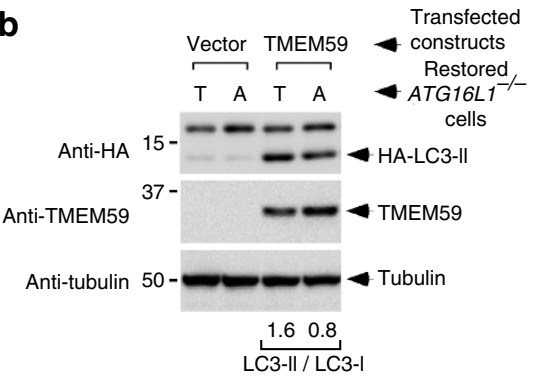

e
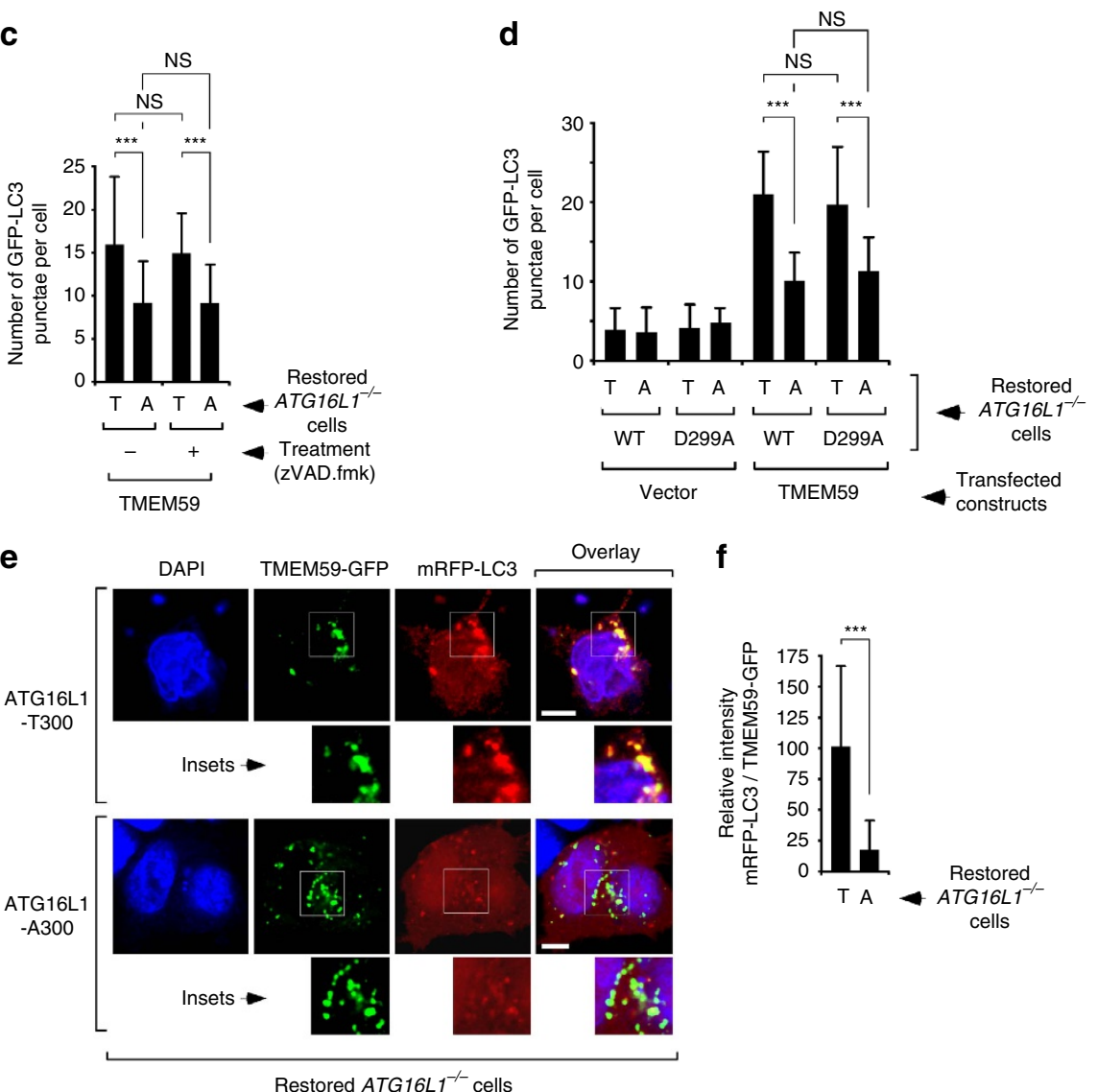

f

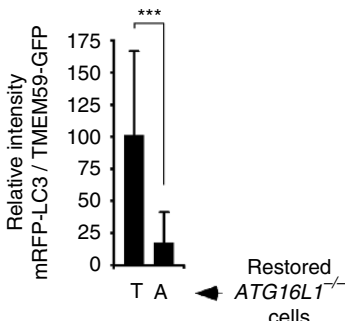

cells

Figure 2 | Defective autophagic function of TMEM59 in the presence of the ATG16L1-A300 Crohn's disease risk allele. (a) Quantification of GFP-LC3 punctae per transfected cell induced by TMEM59 overexpression for 24 $\mathrm{h}$ in ATG16L1-/ - HCT116 cells restored with HA-ATG16L1-T300 (T) or HA-ATG16L1-A300 (A). Shown are mean values \pm s.d. ( $n=50$ cells, ${ }^{\star \star \star} P<0.001$ Student's $t$-test). (b) Immunoblot analysis of HA-LC3 lipidation induced by TMEM59 overexpression in the same conditions and cell lines as in $\mathbf{a}$. The LC3-II/LC3-I signal intensity ratio is shown at the bottom of the relevant lanes. (c,d) Quantification of GFP-LC3 punctae per transfected cell induced by TMEM59 overexpression for $36 \mathrm{~h}$ in the same cell lines as in $\mathbf{a}$, and in the absence or presence of zVAD.fmk (c, $50 \mu \mathrm{M}$ for the last $8 \mathrm{~h}$ of culture), or in ATG16L1-/ - HCT116 cells restored with the indicated ATG16L1 mutants (d). Data are displayed as in a (NS, not significant, $P>0.05$ Student's $t$-test; ${ }^{\star \star \star} P<0.001$ Student's $t$-test). (e) Representative confocal pictures showing colocalization between TMEM59-GFP and mRFP-LC3 co-expressed for $36 \mathrm{~h}$ in the same cell lines as in a. Scale bars represent $5 \mu \mathrm{m}$. (f) Quantification of the intensity of mRFP-LC3 present in TMEM59-GFP-positive vesicles (mean \pm s.d., $n=100$ vesicles present in 8 cells, ${ }^{\star \star \star} P<0.001$ Student's $t$-test; right panel). Data are presented as a fraction of the mean value obtained in cells expressing ATG16L1-T300. All displayed results are representative of at least two independent repetitions.

(Supplementary Fig. 2a) that is insensitive to caspase cleavage (ATG16L1-A300-D299A; Supplementary Fig. 3b) displayed similar autophagic defects compared with caspase-sensitive ATG16L1-A300 (Fig. 2d). Control experiments showed no overt influence of the double mutant on basal or nutritional autophagy (Supplementary Fig. 2d-f). These results indicate that the autophagic defects displayed by TMEM59 in the presence of the risk allele do not require caspase processing of ATG16L1A300.
Our previous studies in a variety of cell lines indicated that the autophagic activity of TMEM59 is directed against the same intracellular vesicles where this protein is located ${ }^{41}$, and this was also the case in HCT116 cells (Fig. 2e). In a series of confocal quantification experiments, we found that the vesicles harbouring TMEM59-GFP were decorated on average with less monomeric RFP (mRFP)-LC3 in HCT116 cells expressing the risk allele compared with those restored with the T300 form (Fig. 2e,f). These results indicate that ATG16L1-A300 impairs the ability of 
TMEM59 to induce the autophagic labelling of its own vesicular compartment.

The A300 allele alters TMEM59 intracellular trafficking. Unexpectedly, we observed that the intracellular trafficking of TMEM59 was altered in cells expressing the A300 allele. Thus, these cells exhibited an increased number of TMEM59-GFPpositive vacuoles compared with those expressing ATG16L1T300, both in HCT116 cells engineered to harbour the T300A mutation (knock-in (KI)-HCT116-A300 (ref. 45); Fig. 3a) and in ATG16L1 ${ }^{-/-}$HCT116 cells restored with HA-ATG16L1-A300 (Fig. 3b). This is likely a loss-of-function phenotype, since
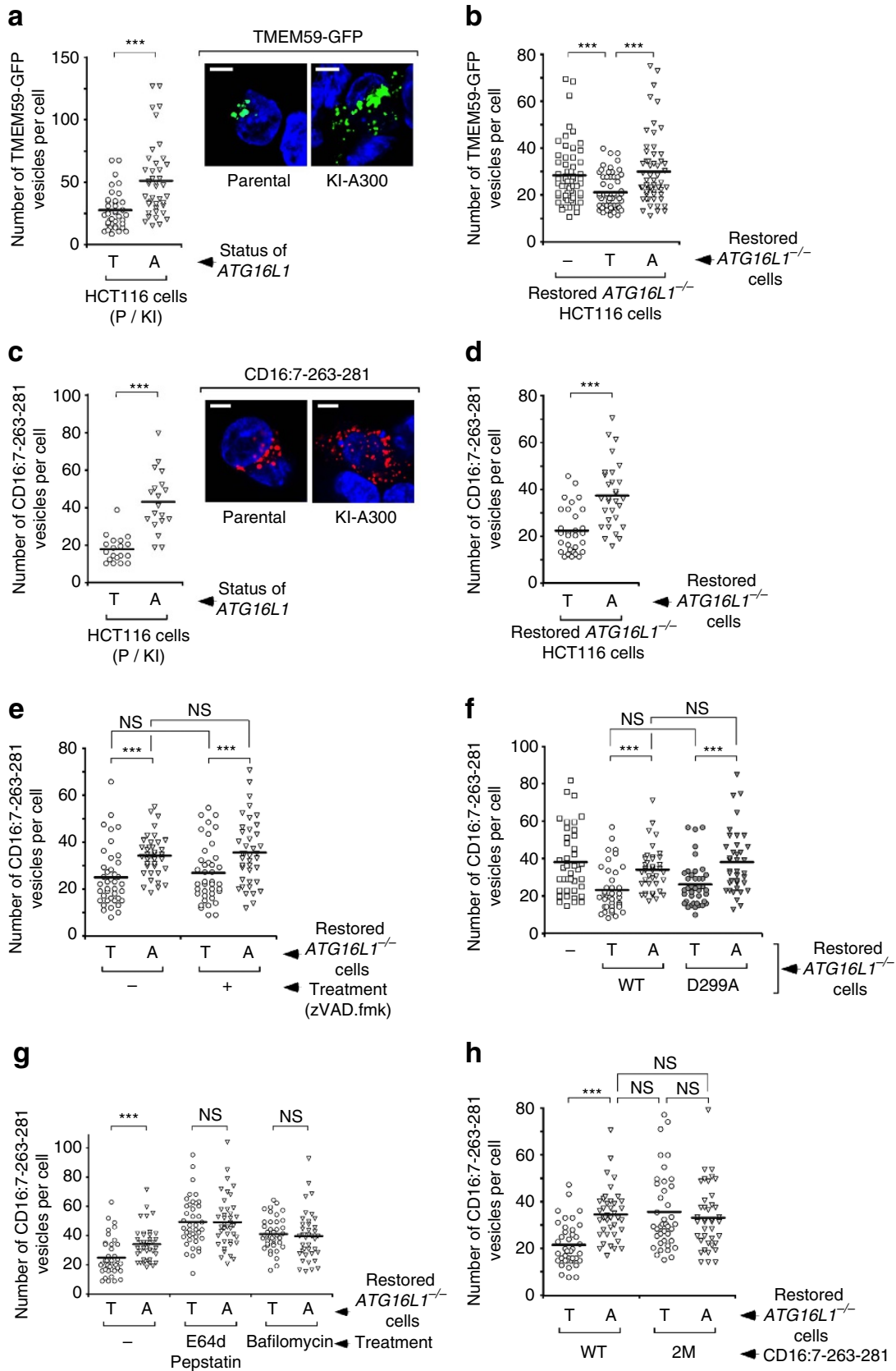

Figure 3 | Altered intracellular trafficking of TMEM59 in cells expressing ATG16L1-A300. (a,b) Quantification of the number of TMEM59-GFP-positive vesicles per cell $24 \mathrm{~h}$ after transfection in parental (T) and ATG16L1-A300 knock-in (KI; A) HCT116 cell lines (a, $n=40$ cells), or in ATG16L1-/ - HCT116 cells restored with T300 (T) or A300 (A) forms of ATG16L1, or irrelevant vector ( - ), (b, $n=60$ cells). Shown are scatter plots where each event represents the score obtained for one cell and the thick horizontal line indicates the mean value $\left({ }^{\star \star \star} P<0.001\right.$ Student's $t$-test). The inset shows representative confocal pictures; scale bars represent $5 \mu \mathrm{m}$. (c,d) Quantification of the number of intracellular vesicles containing aggregated, endocytosed CD16:7-263-281 chimera per cell after aggregation with anti-CD16 antibodies in the cellular strains shown in $\mathbf{a}$ and $\mathbf{b}$, respectively (c, $n=20$ cells; $\mathbf{d}, n=30$ cells). ${ }^{\star \star \star} P<0.001$ Student's $t$-test. The inset shows representative confocal pictures; scale bars represent $5 \mu \mathrm{m}$. (e-h) Quantification of the number of intracellular vesicles containing aggregated, endocytosed CD16:7-263-281 chimera in ATG16L1-/- HCT116 cells restored with the indicated ATG16L1 constructs, (e) in the absence or presence of zVAD.fmk ( $50 \mu \mathrm{M}$, last $8 \mathrm{~h}$ of culture; $n=40$ cells), (f) in cells restored with caspase-3-sensitive and -insensitive (D299A) ATG16L1 constructs ( $n=40$ cells), $(\mathbf{g})$ in the absence or presence of E64d/pepstatin (10 $\mu \mathrm{g} \mathrm{ml}{ }^{-1}$ each, $\left.8 \mathrm{~h}\right)$ or bafilomycin ( $50 \mathrm{nM}$, $8 \mathrm{~h} ; n=40$ cells) or (h) after aggregation of wild type or Y268A,E272A (2M) versions of the CD16:7-263-281 chimera $\left(n=40\right.$ cells). ${ }^{\star \star \star} P<0.001$ Student's t-test; NS, not significant, $P>0.05$ Student's $t$-test. All shown results are representative of at least two independent repetitions. 
ATG16L1-1- cells displayed the same anomaly (Fig. 3b). A similar defect was observed on forced aggregation of a chimeric transmembrane molecule containing the minimal active element of TMEM59 (CD16:7-263-281) ${ }^{41}$ with anti-CD16 antibodies (Fig. 3c,d), indicating an involvement of the ATG16L1-binding peptide. This result also argues that the trafficking alteration primarily affects the endocytic route, not the maturation pathway that exports TMEM59 from the endoplasmic reticulum to the plasma membrane. Again, neither zVAD.fmk (Fig. 3e) nor ATG16L1-A300-D299A (Fig. 3f and Supplementary Fig. 4a) prevented this phenotype, indicating that it does not require caspase processing of the A300 allele.

Given our previous results showing that TMEM59-positive endosomes recruit ATG16L1 and become labelled with LC3 to be more efficiently targeted for lysosomal degradation ${ }^{41}$, we investigated whether the accumulation of vesicles in the presence of the risk allele was due to a slower transit through the endocytic route. Treatment with lysosomal inhibitors normalized the number of vacuoles carrying CD16:7-263-281 (Fig. 3g) or TMEM59-GFP (Supplementary Fig. 4b) in cells expressing ATG16L1-T300 compared with those harbouring the risk form, suggesting that the observed accumulation was not due to an increased supply of vesicles to the endocytic route (that is, enhanced endocytosis) promoted by the A300 allele. In addition, a mutated version of the motif that blocks the autophagic activity but leaves the embedded YXXL endocytosis motif intact $(\mathrm{Y} 268 \mathrm{~A}, \mathrm{E} 272 \mathrm{~A} ; 2 \mathrm{M})^{41}$ recapitulated the defective trafficking phenotype of CD16:7-263-281 in the presence of the T300 non-risk form (Fig. 3h). Conversely, it had no effect in ATG16L1A300-expressing cells (Fig. $3 \mathrm{~h}$ ), arguing that the double mutation (2M) and ATG16L1-A300 cause redundant alterations. Together, these results suggest that poor LC3 labelling of the endosomes where TMEM59 is located slows down their transit along the endocytic route in cells expressing ATG16L1-A300, thus causing the observed vesicle accumulation. In line with this idea, the expression levels of transfected TMEM59 were often higher in the presence of defective ATG16L1, whether this defect came from uncoupling the $\mathrm{N}$ - and C-terminal domains of the molecule (see TMEM59 immunoblot in Fig. 1a) or was caused by the T300A mutation (Fig. 2b).

Defective binding of ATG16L1-A300 to TMEM59. Since the functional alterations introduced by ATG16L1-A300 in the autophagic activity and trafficking of TMEM59 are not prevented by caspase- 3 inhibition (Figs $2 \mathrm{c}$ and $3 \mathrm{e}$ ) or expression of a caspase-3-insensitive ATG16L1-A300 construct (ATG16L1A300-D299A; Figs 2d and 3f and Supplementary Fig. 4a), they likely result from pathological mechanisms other than the reported increased susceptibility of the risk allele to caspase cleavage ${ }^{46,47}$. One possibility is that the A300 polymorphism alters the ability of the WDD to interact with its target motif. To test this idea we performed co-immunoprecipitation studies after expression of the relevant partners. We found that TMEM59 tagged with glutathione-S-transferase (GST; TMEM59-GST) coprecipitated less efficiently with the A300 variant of full-length ATG16L1 in transfected HEK-293T cells (Fig. 4a). The detected interaction was mediated by the WDD-binding motif, since it was inhibited by mutation of the four critical residues (4M version; Fig. 4a). In addition, less TMEM59 was found in GST-ATG16L1A300 precipitates (Fig. 4b). Similar results were obtained in HCT116 cells engineered by knock-in to express ATG16L1-A300, and in Atg16l1 ${ }^{-1}-$ mouse embryonic fibroblasts (MEFs) ${ }^{36}$ or ATG16L1 ${ }^{-1}$ HCT116 cells restored with T300 or A300 versions of ATG16L1 (Fig. 4c). Restored MEFs showed comparable levels of both ATG16L1 forms (Supplementary
Fig. 5a), and unaltered basal autophagy in the presence of ATG16L1-A300 (Supplementary Fig. 5b). These data suggest that the T300A mutation impairs the ability of the WDD to interact with its natural ligands. However, given that the intracellular trafficking of TMEM59 is abnormal in ATG16L1-A300expressing cells (Fig. 3), such impaired binding might be explained by an altered subcellular localization of any of the partners (TMEM59-GST or HA-ATG16L1 itself). To clarify this issue, we resorted to in vitro systems based on peptide arrays or pull-down co-precipitation assays involving recombinant proteins.

We designed peptide microarrays in which the 19-amino acid peptide of TMEM59 that includes the ATG16L1-binding motif (263-281) was immobilized onto glass slides along with point mutants as controls. A subset of the peptides included a biotin group introduced at the end of the synthesis procedure to allow assessment of the amount of immobilized peptide. We probed the resulting arrays with a recombinant form of the WDD fused to GST and the HA tag (GST-HA-WD-320-607) purified from bacterial expression systems, or an extended WDD construct that includes residue 300 (GST-HA-ATG16L1-231-607, excluding the $\mathrm{N}$-terminal and coiled-coil domains ${ }^{27}$ produced in yeast. Both proteins were able to recognize the wild-type peptide but reacted poorly with the $4 \mathrm{M}$ derivative (Fig. 5a,b), suggesting that the observed interaction involves the WDD-binding motif. These results obtained with ligands produced in heterologous systems argue that the interaction with the target peptide is probably direct, since it is unlikely that a contaminating protein from bacteria or yeast (whose Atg16 ortholog lacks the WDD) mediates the binding event. Notably, a ligand preparation purified from mammalian expression systems showed superior binding activity (Fig. 5c), perhaps due to post-translational requirements provided by a native environment. Again, this interaction was motif-specific, since it was largely abrogated by mutation of the critical residues ( $4 \mathrm{M}$ version; Fig. $5 \mathrm{c}$ ). None of the observed differences was due to dissimilar amounts of immobilized peptides, because an anti-biotin antibody reacted equally well with the relevant peptides (Fig. 5d). In addition, we detected reduced binding between GST-HA-ATG16L1-231-607 (produced in human cells) and peptides harbouring mutations in functionally important positions (Y268, Y277 and L280) ${ }^{41}$, but not in the case of mutants involving irrelevant residues such as V269, K273 or G278 (ref. 41; (Fig. 5e)). One notable exception was E272A, which showed normal binding to the WDD ligand (Fig. 5e) but, according to our previous studies, is critical for LC3 lipidation ${ }^{41}$. Interestingly, we also showed that this mutation does not inhibit recruitment of GFP-LC3 to TMEM59 (ref. 41), suggesting that E272 is exclusively involved in the LC3 lipidation step but not in recruitment of LC3-I to the lipidating complex ${ }^{41}$, a step known to be mediated by ATG16L1 through ATG5 and ATG3 (ref. 50). Therefore, an involvement of this residue in binding to ATG16L1 should not be expected in the peptide array setting. Together, these data argue that the observed interaction between the extended WDD ligand (residues 231-607) and the immobilized target peptides faithfully reflects the functional features of the 263-281 element.

We next used ligand preparations purified from human cells to test whether the T300A mutation alters the ability of the WDD to interact with the motif in this system. Binding assays conducted with GST-HA-ATG16L1-231-607 in a T300 or A300 configuration showed that the latter recognized the native peptide with a significantly lower affinity compared with the T300 form (Fig. 5f). The levels of background signal measured using a variety of methods were similar in the arrays developed with both ligand versions (Fig. 5g), indicating that the assay was performed under comparable conditions in both cases. Consistent with these 

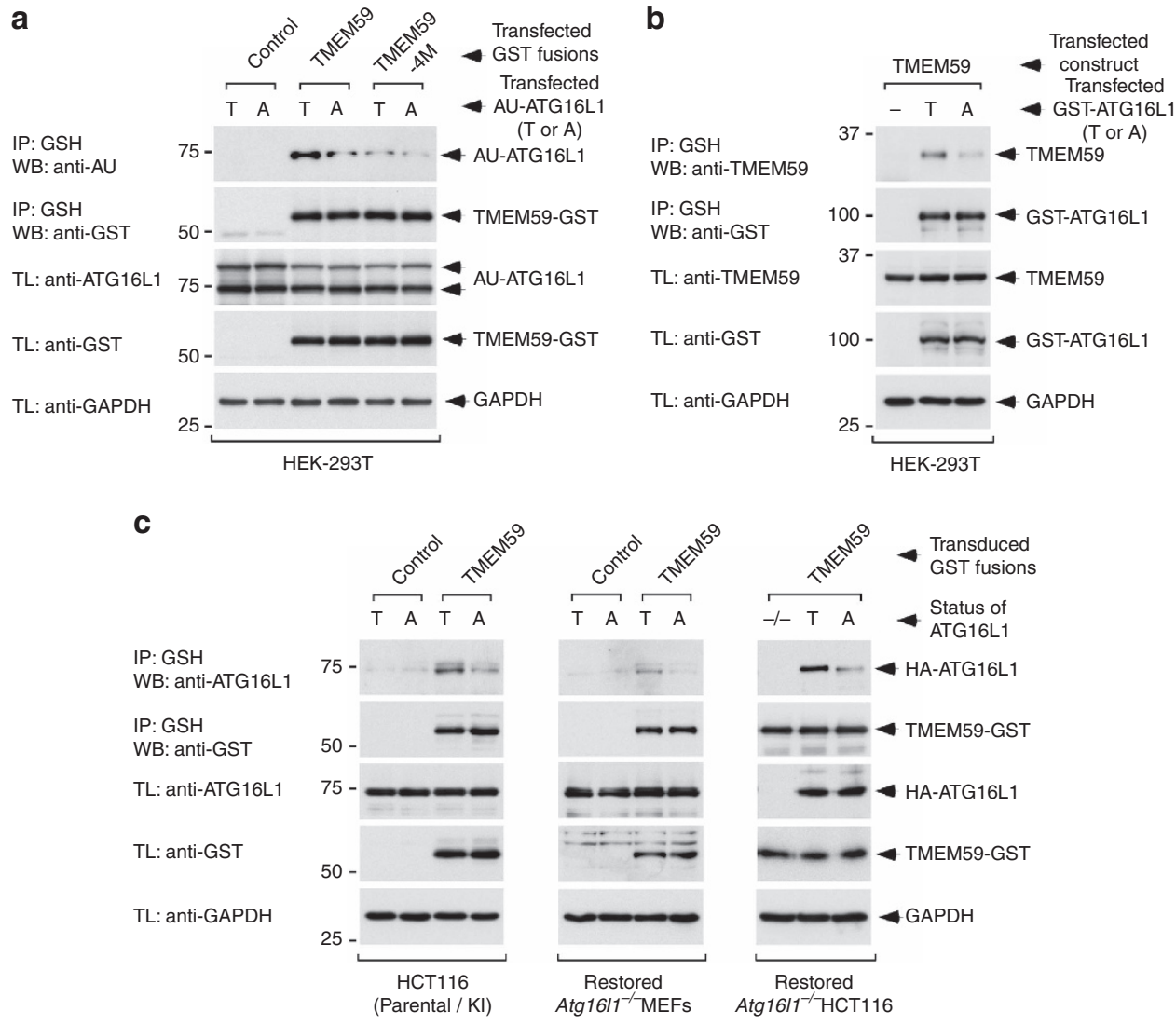

Figure 4 | Impaired binding between TMEM59 and ATG16L1-A300 in co-precipitation assays. (a,b) Immunoblot analysis of agarose-glutathione (GSH) immunoprecipitates (IP) or total cell lysates (TL) from HEK-293T cells transfected with the indicated constructs and lysed $36 \mathrm{~h}$ after transfection. (c) Immunoblot analysis of GSH immunoprecipitates (IP) or whole-cell lysates (TL) of the indicated cell strains retrovirally transduced with TMEM59-GST for $36 \mathrm{~h}$. All displayed results are representative of at least three repetitions.

results, pull-down studies carried out with fusion proteins between GST and the active element (GST-263-281; expressed and purified from bacteria) showed that full-length ATG16L1A300 binds defectively to the target peptide (Fig. 5h). The interaction defect was more profound in this system compared with the data obtained using peptide microarrays (Fig. 5f), perhaps because the mutation alters ATG16L1 folding more severely in heterologous environments. Together, these data suggest that the T300A mutation causes an intrinsic structural alteration in the WDD that disrupts its ability to bind the ATG16L1-binding motif that we previously identified in TMEM59.

Certain WDD-binding motif versions are insensitive to T300A. To determine whether this disability prevents binding of the WDD to alternative versions of the motif, we tested other peptides harbouring different configurations of the pattern for binding to both forms of ATG16L1. We previously identified four peptides of varying length present in unrelated proteins $\left(\mathrm{DEDD} 2_{12-25}, \mathrm{~T}^{3} \mathrm{JAM}_{318-333}, \mathrm{NOD} 2_{63-78}\right.$ and TLR2 $\left.2_{761-779}\right)$ that are able to bind the WDD of ATG16L1 (ref. 41). Regardless of whether these elements play an ATG16L1-binding role in the context of their native proteins, when isolated they constitute alternative members of the ATG16L1-binding motif landscape ${ }^{41}$. Pull-down studies carried out with fusion constructs between GST and the different peptides showed that each was sufficient to bind ATG16L1-T300 expressed in bacteria (Fig. 6), thus confirming that they harbour an ATG16L1-binding signature. However, in two cases (DEDD2 and T3JAM), binding to the
A300 form was blunted (Fig. 6), whereas the other two (TLR2 and NOD2) bound equally well (or better) to the $\mathrm{CD}$ risk protein (Fig. 6). Therefore, the disruption introduced by the T300A mutation in the ability of the WDD to bind its peptidic partners is restricted to particular versions of the ATG16L1-binding motif.

Defective xenophagy by disruption of the TMEM59-ATG16L1 axis. We previously showed that, on infection with $S$. aureus, TMEM59 binds ATG16L1 to induce an unconventional autophagic event that promotes LC3 labelling of bacteria-containing phagosomes ${ }^{41}$. To test whether this interaction is altered by the T300A mutation, we carried out co-precipitation studies in cells infected with S. aureus. In this case we used Atg16l1-/- MEFs restored with T300 or A300 versions of HA-ATG16L1, since they showed a stronger autophagic response to $S$. aureus compared with HCT116 cells (Supplementary Fig. 6). We found that, at early infection time points ( $2 \mathrm{~h}$ post-infection), TMEM59 associated defectively with ATG16L1-A300 compared with the T300 form (Fig. 7a). No major differences in infection rates were detected between the two cellular strains, as indicated by the levels of GFP (which is constitutively expressed by the bacteria) present in infected cells (Fig. 7a). Notably, we found no decay in the levels of full-length ATG16L1 (Supplementary Fig. 7) and no evidence of cleaved ATG16L1 or caspase-3 activation (Supplementary Fig. 7) in the same samples, arguing that the interaction defect does not require caspase-3-mediated processing of ATG16L1-A300.

The binding impairment displayed by the A300 allele correlated with reduced LC3 lipidation in response to $S$. aureus 
infection (Fig. 7a), decreased labelling of bacterial phagosomes with LC3 (Fig. 7b) and increased recovery of colony-forming units from infected cells (Fig. 7c), indicating that the T300A mutation causes defective xenophagy and reduced control of the infection at an early stage. As expected, Atg16l1-/- MEFs showed poor xenophagy in reaction to the bacteria (Fig. 7d-f). Expression of non-risk ATG16L1 restored this response (Fig. 7d-f), part of which was lost in cells expressing separated

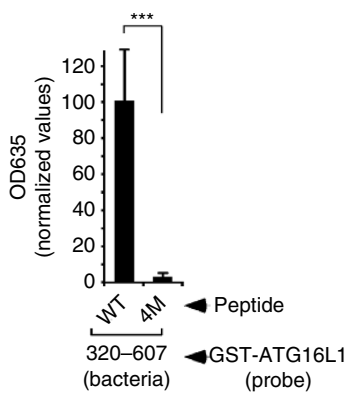

b

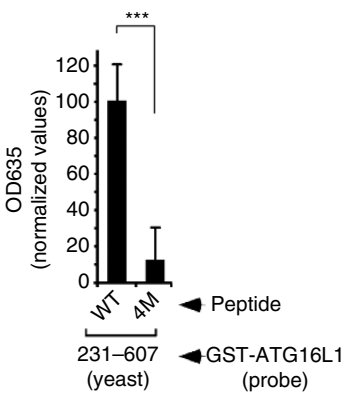

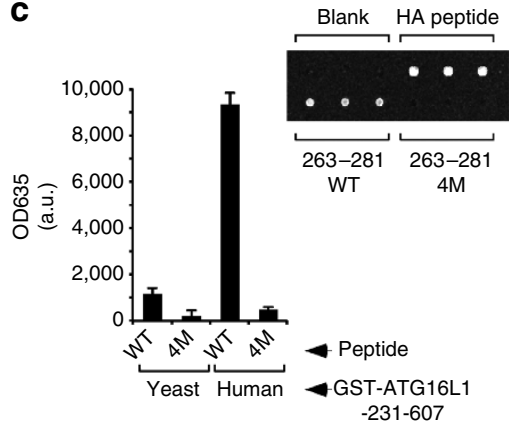

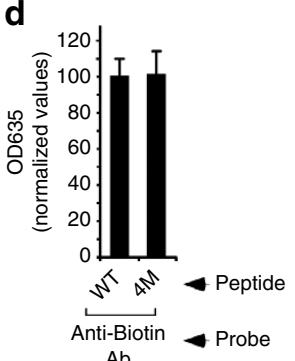

$\mathrm{Ab}$

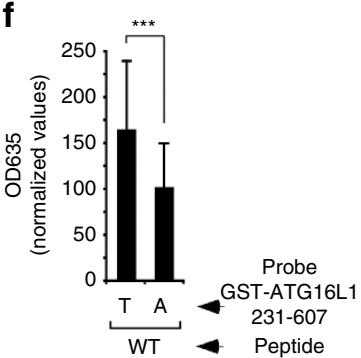

$\mathbf{e}$
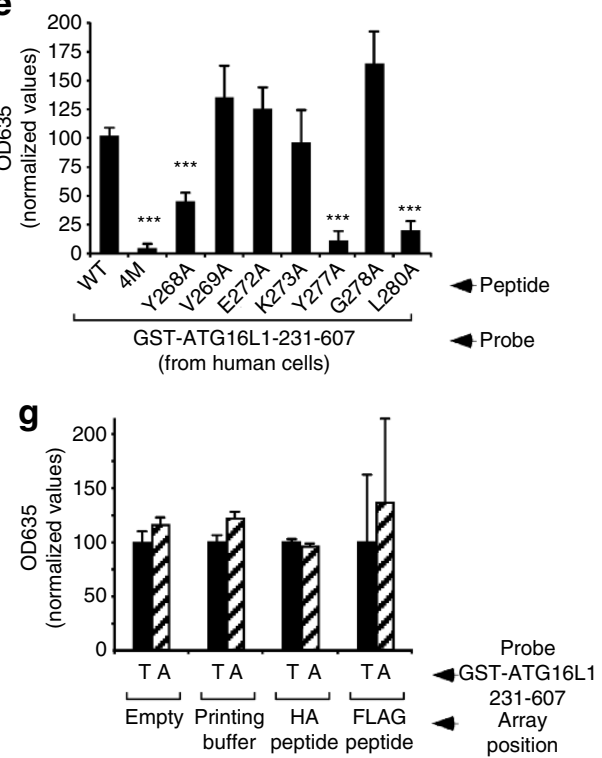
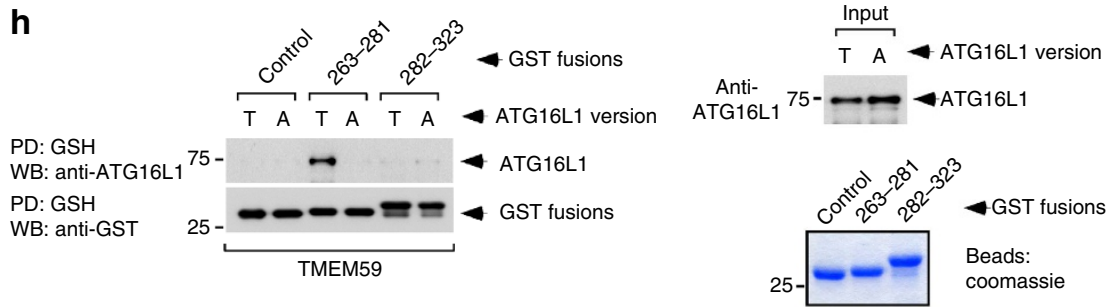

Figure 5 | Defective in vitro binding between the active peptide 263-281 and ATG16L1-A300. (a-g) Quantification of binding signals provided by peptide microarrays developed with different ligands. Figures show mean values \pm s.d. $\left(n=9\right.$ replicas, ${ }^{\star \star \star} P<0.001$ Student's $t$-test), unless otherwise indicated. (a,b,d,e) Values are expressed as a fraction of the figures provided by the wild type (WT) peptide. (a) Binding of GST-ATG16L1-320-607 expressed in bacteria to WT or 4M versions of the 263-281 peptide. (b) Binding of GST-ATG16L1-231-607 purified from yeast to WT or 4M versions of the 263-281 peptide. (c) Binding of GST-ATG16L1-231-607 purified from yeast or human cells (as indicated) to WT or 4M versions of the 263-281 peptide. The inset shows a magnified scanned image of an actual peptide microarray developed with the ligand purified from human cells. (d) Binding of an anti-biotin antibody to WT or 4M versions of the 263-281 peptide. (e) Binding of GST-ATG16L1-231-607 purified from human cells to the indicated mutant derivatives of the 263-281 peptide. (f) Binding of GST-ATG16L1-231-607 in T300 (T) or A300 (A) configurations (as indicated) purified from human cells to the wild-type 263-281 peptide (WT). Values are expressed as a fraction of the figures provided by the A300 ligand $(n=54)$. (g) Background binding of GST-ATG16L1-231-607 (T300 or A300 variants, as shown) purified from human cells to the indicated irrelevant array positions. No subtraction of background signal was done in this case. Values are expressed as a fraction of the figures provided by the T300 ligand version. (h) Immunoblot analysis of pull-down assays (PD) between the indicated GST fusion proteins expressed in bacteria and full-length ATG16L1-T300 (T) or -A300 (A) (as indicated) present in crude bacterial lysates (left panel). The right panel shows an immunoblot assay for the presence of ATG16L1 (T or A) in the bacterial lysates before the assay was done (input, top), and a Coomassie-blue staining of a protein gel including the different GST fusion proteins (bottom). The 282-323 construct includes the non-functional part of the intracellular domain of TMEM59. All displayed results are representative of at least two repetitions. 


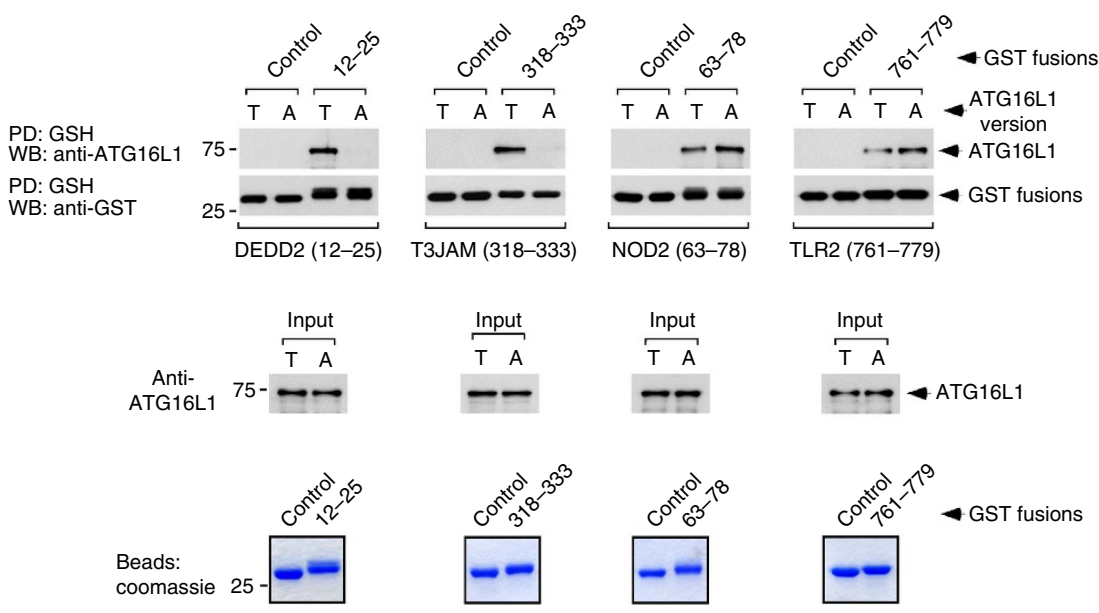

Figure 6 | Differential impact of T300A on the interaction between ATG16L1 and different versions of the WDD-binding motif. Immunoblot analysis of pull-down assays (PD) between the indicated GST fusion proteins purified from bacteria and ATG16L1-T300 (T) or ATG16L1-A300 (A) present in crude bacterial lysates (top panels). The middle panels show immunoblot assays for the presence of ATG16L1 (T or A) in the bacterial extracts before the assay was carried out (input). The bottom panels show Coomassie blue-stained protein gels including the different purified GST fusion proteins used for the pull-down studies. The results shown are representative of at least three repetitions.

N- and C-terminal ATG16L1 fragments (Fig. 7d-f). These data indicate a role of ATG16L1 in restraining S. aureus viability, and show that a portion of such activity is mediated by the WDD. Consistent with a function of TMEM59 in this pathway, depletion of this molecule using short interfering RNAs (siRNAs) recapitulated the xenophagic defects observed in the presence of ATG16L1-A300 or split ATG16L1 (Supplementary Fig. 8a-d). The cellular strains analysed did not show substantial differences in the number of intracellular GFP-positive bacteria (Supplementary Fig. 9), suggesting comparable infection rates. Together, these results point to the existence of an early xenophagic burst mediated by the WDD that is derailed by the A300 allele, at least in part through an inability of this mutant to bind the ATG16L1-binding motif. A fraction of such response is likely induced by TMEM59, although other molecules including the motif could also be involved. Importantly, cells expressing split ATG16L1 retained some xenophagic activity (Fig. 7d-f), suggesting that an overlapping layer of WDD-independent, conventional autophagy may also contribute to this function.

\section{Discussion}

Since the discovery of the T300A polymorphism as a risk factor for CD (refs 6,7), a substantial body of work has revealed a role of ATG16L1 in a variety of processes linked to intestinal homeostasis, including xenophagy against invading microorganisms ${ }^{36,39,40}$, trafficking of secretory vesicles in intestinal cells ${ }^{36,38}$ or the control of inflammation ${ }^{34,35}$. Additional work has demonstrated that many of these processes are also altered in the presence of the A300 allele ${ }^{34,38-40,45-49,51}$. The recent discovery that the T300A mutation increases susceptibility of ATG16L1 to caspase processing ${ }^{46,47}$ has provided a major advance towards identification of the molecular dysfunctions that cause these alterations. Thus, under stressful conditions such as bacterial infection or starvation, caspase-3 preferentially cleaves the risk allele and reduces the levels of the full-length protein, leading to impaired autophagy, decreased xenophagy and increased production of inflammatory mediators ${ }^{46,47}$.

We now reveal that the T300A mutation also derails the normal function of ATG16L1 in the absence of caspase-3mediated cleavage. This single amino acid change alters the ability of the C-terminal WDD to interact with an amino acid motif that we previously identified in the transmembrane protein TMEM59 (ref. 41). In the context of this molecule, the mutation impairs binding between the WDD and the motif, a defect that results in poor autophagic labelling of the endosomes in which TMEM59 is located and slowed transit of these vesicles through the endocytic route. However, additional versions of the motif also displayed binding defects to the risk allele (see for example the motifs present in T3JAM and DEDD2; Fig. 6), suggesting that a cohort of ATG16L1 activators containing different configurations of the pattern have their activities blunted by the mutant protein in non-stressed conditions. Members of this family that may be involved in xenophagy (like TMEM59) could facilitate the onset of the uncontrolled inflammatory response that is typical of $\mathrm{CD}$ by being unable to restrain intracellular proliferation of the pathogens in the presence of the risk allele. However, our findings suggest alternative mechanisms through which ATG16L1-A300 could contribute to inflammation. For instance, expression of T3JAM and DEDD2 has been shown to increase c-Jun Nterminal kinase (JNK) signalling ${ }^{52}$ and apoptosis ${ }^{53}$, respectively, and both the JNK pathway ${ }^{54}$ and programmed cell death ${ }^{55}$ are thought to participate in intestinal inflammation. It is possible that an impaired interaction with ATG16L1-A300 (Fig. 6) could lead to decreased autophagic degradation and, as a consequence, increased levels of these mediators, thus promoting excessive activity of these signalling routes in the face of certain stimuli. Interestingly, we found that the interaction between the WDD and different motif versions is dissimilarly affected by the T300A mutation, arguing that some WDD-binding proteins might actually function better in the presence of the risk allele, or may not be affected at all. These results suggest a complex picture of the activity of the motif in the presence of ATG16L1-A300.

We show here that the A300 risk allele alters two cellular functions reminiscent of the defects observed in $\mathrm{CD}$ experimental model systems. First, ATG16L1-A300 impairs the normal intracellular trafficking of endosomes harbouring TMEM59. Derailed trafficking of secretory vesicles and a disorganized secretory compartment are well-known phenotypes of the intestinal Paneth and goblet cells of both mice and CD patients carrying the ATG16L1-A300 allele ${ }^{38,46}$. It is conceivable that a key regulator of these specialized vesicles also includes the motif and its dysfunction in the presence of the risk allele alters 
a

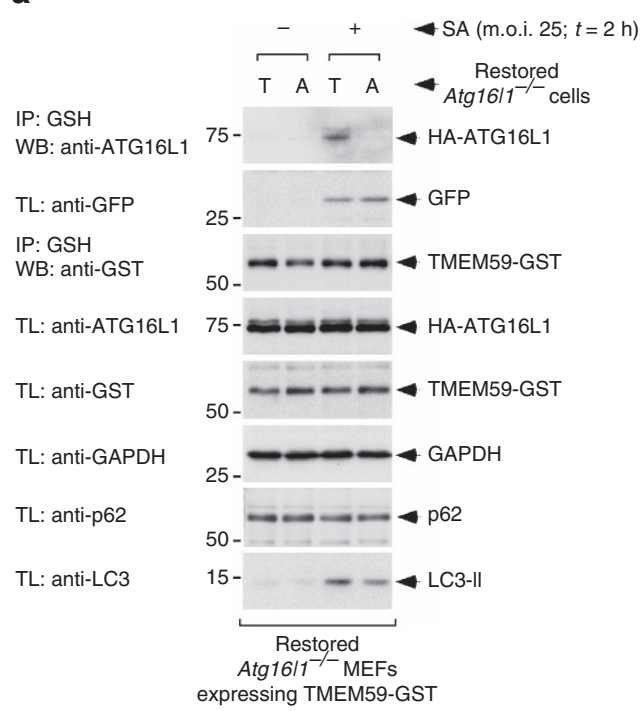

b

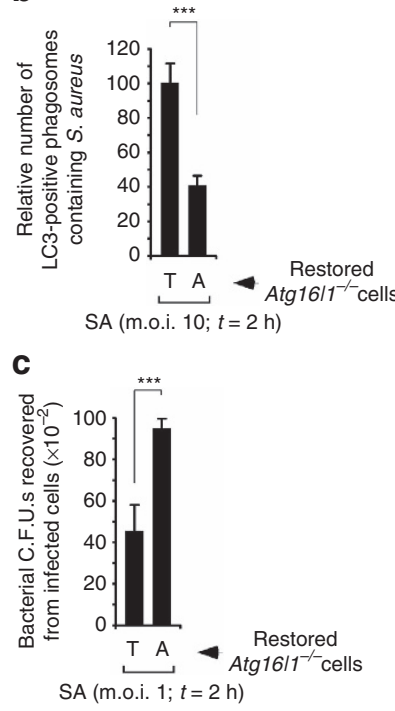

d

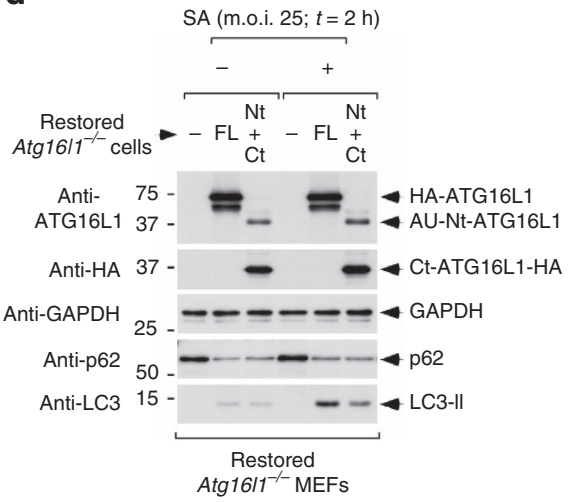

e

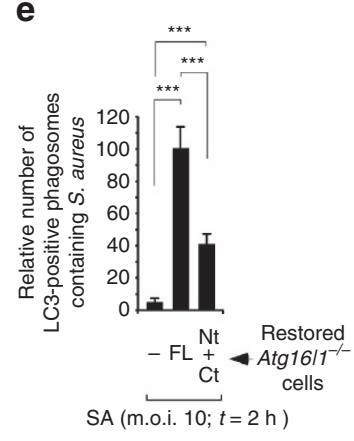

f

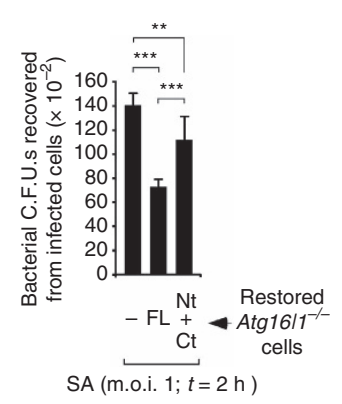

Figure 7 | ATG16L1-A300 binds defectively to TMEM59-GST and impairs xenophagy in response to S. aureus. (a) Immunoblot analysis of GSH immunoprecipitates (IP) or total cell lysates (TL) of Atg16/1-/- MEFs reconstituted with the indicated versions of ATG16L1 (T300 (T) or A300 (A)), retrovirally transduced with TMEM59-GST and subsequently infected $(+)$ with a S. aureus strain (SA; 2 h; multiplicity of infection (m.o.i.) $=25)$ that constitutively expresses GFP, or left uninfected $(-)$. These results are representative of three repetitions. (b,e) Quantification of the number of LC3-positive phagosomes containing GFP-positive S. aureus bacteria $2 \mathrm{~h}$ after infection (m.o.i. =10) in Atg16L1-/- MEFs restored with the indicated versions of ATG16L1 (T or A), full-length ATG16L1-T300 (FL), both ATG16L1 fragments that result from caspase-3 processing of the risk allele (Nt: 1-299; Ct: 300-607) or irrelevant vector ( - ). Values are expressed as a fraction of the scores obtained for cells expressing ATG16L1-T300 (mean \pm s.d. of triplicates; $n=500$ cells; ${ }^{\star \star \star} P<0.001$ Student's $t$-test). (c,f) Quantification of the colony-forming units (C.F.U.s) recovered from Atg16L1- $/-$ MEFs restored with the indicated versions of ATG16L1 (T or A) and infected with S. aureus for $2 \mathrm{~h}$, m.o.i. $=1$ (mean \pm s.d., $n=6,{ }^{\star \star \star} P<0.001$, ${ }^{\star \star} P<0.01$ Student's t-test). (d) Immunoblot analysis of endogenous LC3 activation and p62 expression levels induced by S. aureus infection ( $2 \mathrm{~h}, \mathrm{~m} .0 . \mathrm{i} .=25)$ of Atg16/1- $/-$ MEFs reconstituted with the indicated versions of ATG16L1 (full-length ATG16L1-T300 (FL) or both ATG16L1 fragments that result from caspase-3 processing of the risk allele (Nt: 1-299; Ct: 300-607)), or control vector (-). Results are representative of at least two repetitions.

their proper secretory trafficking. Second, we found that ATG16L1-A300 derails an early WDD-dependent xenophagic response against $S$. aureus that contributes to fight the infection. Conflicting results have been published regarding the overall role of autophagy in $S$. aureus infection, ranging from no effect ${ }^{56}$ to a supporting role in bacterial replication ${ }^{41,57}$. Notably, we show here that ATG16L1 induces a concomitant layer of WDD-independent xenophagy in response to S. aureus (Fig. 7). A complex interplay between these two overlapping xenophagic activities, together with the varying intensity of basal, conventional autophagy in different cellular systems, and the known role of this process in favouring $S$. aureus replication at later stages ${ }^{41,57}$, might account for such different observations. In addition, ATG16L1 has been shown to promote tolerance to $S$. aureus $\alpha$-toxin ${ }^{56}$, an activity that could be explained by the central function of autophagy in increasing resistance to stressful situations. But regardless the complexity of this particular system, the defects in WDD function caused by the T300A mutation that we describe here provide a novel mechanistic view to understand the more general alterations in xenophagy previously linked to this allele and CD (refs 39,48,49). Interestingly, the A300 polymorphism occurs at high frequency in the human population $^{6,7}$, and it has been associated with additional physiopathological situations, like decreased survival following bone marrow transplantation ${ }^{58}$, improved survival in colorectal cancer $^{59}$, gastric cancer susceptibility ${ }^{60}$ or Paget's disease of bone $^{61}$. Therefore, understanding the biochemical consequences of the T300A mutation might be an important first step towards unravelling the physiological role of this allele beyond CD.

Our data support that the unconventional autophagic activity of WDD-binding proteins is also blunted in the presence of caspase-3-mediated cleavage of ATG16L1-A300, since TMEM59 
was unable to trigger LC3 activation in a cellular system in which the entire pool of ATG16L1 was uncoupled in two fragments (thus mimicking caspase-mediated cleavage). In contrast, basal and rapamycin-induced autophagy were unaffected in these extreme conditions. This result is consistent with previous reports showing that the WDD is not involved in conventional autophagy ${ }^{28-31}$. However, other reports have found inhibition of the canonical pathway by cleaved ATG16L1 (refs 46,47). These discrepancies suggest that, in some experimental systems, the WDD might play a role in conventional autophagy, or that under certain conditions the canonical route may include a relevant component of WDD-mediated unconventional autophagic processes similar to the one induced by TMEM59.

Our findings imply that a single amino acid change (T300A) is able to cause two different outcomes: altered affinity of the WDD for its target motif and increased susceptibility to caspase-3 processing. Although an alanine residue in position $\mathrm{P1}^{\prime}$ of a consensus site is known to enhance caspase- 3 processing of synthetic peptides ${ }^{62}$, the T300A mutation could also promote a shift in the overall structure of the WDD that might improve accessibility of the site to caspase 3 . In this scenario, both effects could be the consequence of the same structural alteration imposed by the T300A mutation.

Together, our results help draw a coherent molecular model regarding how the A300 polymorphism causes pathology. We propose that a collection of motif-containing WDD-binding proteins have their natural autophagic activities modified by the risk allele under homeostatic situations in which levels of caspase 3 activity are negligible. In addition, the activity of these effectors would be blunted by ATG16L1-A300 processing in biological contexts in which stress promotes caspase- 3 activation. Either way, alterations in the pathway involving motif-containing proteins and the WDD appear to be a prominent consequence of the ATG16L1-A300 polymorphism. Identification of additional members of this family of effectors will likely help unravel the signalling pathways whose dysfunction is required to trigger the onset of CD.

\section{Methods}

Cell lines and reagents. HEK-293T cells were obtained from the American Type Culture Collection. The HCT116 cell line derivatives KI-ATG16L1-T300A (generated by knock-in gene targeting) and $A T G 16 L 1^{-/-}$have been described elsewhere ${ }^{45}$. MEFs deficient in Atg16l1 have been previously described ${ }^{36}$. Cells were cultured at $37^{\circ} \mathrm{C}$ and a humidified $5 \% \mathrm{CO}_{2}$ atmosphere in DMEM (Invitrogen) containing $10 \%$ heat-inactivated FBS (Invitrogen) and $100 \mathrm{U} \mathrm{ml}^{-1}$ penicillin/ streptomycin (Invitrogen). Bafilomycin, E64d, pepstatin, rapamycin, TNF $\alpha$ and cycloheximide were purchased from Sigma. zVAD.fmk was obtained from Calbiochem.

DNA constructs. DNA constructs expressing HA-tagged human ATG16L1 $\beta$ (for mammalian and bacterial expression), HA-LC3, GFP-LC3, TMEM59 (full-length or $\Delta 282$, wild-type or $4 \mathrm{M}$, untagged or C-terminal GST-tagged), p62-HA and CD16:7-263-281 have been previously described ${ }^{41}$. mRFP-LC3A was generated from the GFP-LC3A construct by replacing the GFP open reading frame with a PCR product encoding mRFP previously amplified from the mRFP-N1 plasmid (Addgene \#54635). Cloning sites were Hind3/Pcil. TMEM59-GFP was built by inserting an in-frame PCR product encoding TMEM59 upstream GFP (Hind3/ Pci1). ATG16L1 C- and N-terminal deletions were generated by PCR and insertion of the relevant amplicons downstream (Pcil/Not1) and upstream (EcoR1/Bsph1) $\mathrm{HA}$ and AU tags, respectively. GST fusions with the different peptides harbouring an ATG16L1-binding motif (TLR2 ${ }_{761-779}$, DEDD2 $_{12-25}$, T3JAM ${ }_{318-333}$ and

NOD $2_{63-78}$ ) used for pull-down studies were constructed by PCR and subsequent insertion downstream, the GST open reading frame (EcoR1/Not1). ATG16L1T300A, D299A, T300A/D299A and CD16:7-263-281-Y268A,E272A (2M) mutants were generated by site-directed mutagenesis (QuikChange, Stratagene). The sequences of the relevant oligonucleotides used to create these constructs are shown in Supplementary Tables 1 and 2. For expression in mammalian cells, constructs were cloned into the pEAK series of mammalian expression vectors or a retroviral derivative (P12-MMP) ${ }^{63}$ that includes internal ribosome entry site (IRES)-antibiotic resistance cassettes for selection in the appropriate selective media (see below). For expression in bacteria, GST fusion constructs were cloned into the pGEX vector (GE Healthcare) and ATG16L1 was expressed from the pET plasmid (Novagen). All constructs were verified by sequencing.

Transfections and retroviral transductions. Overexpression and intracellular trafficking experiments were performed by transfection of the relevant DNA constructs using the jetPEI lipid reagent (Polyplus) following the manufacturer's instructions. KI-HCT116 showed lower transfection efficiency when compared to its parental counterpart; for this reason, many of the experiments were conducted in $A T G 16 L 1^{-1-}$ cells restored with T300 or A300 HA-ATG16L1. ATG16L1deficient cells (MEFs and HCT116) were restored with the different ATG16L1 constructs by retroviral transduction. Retroviral transductions were also used to express TMEM59-GST in the indicated experiments. The relevant constructs were subcloned into P12-MMP vector versions including IRES-puromycin (to express full-length ATG16L1 or its N-terminal fragment) or IRES-hygromycin (to express the C-terminal fragment of ATG16L1 or TMEM59-GST) resistance cassettes that allow selection in puromycin $\left(1 \mu \mathrm{g} \mathrm{ml}^{-1}\right)$ and hygromycin $\left(200 \mu \mathrm{g} \mathrm{ml}^{-1}\right)$, respectively. Virus-containing supernatants were generated by co-transfecting $293 \mathrm{~T}$ cells with the relevant P12-MMP constructs together with helper plasmids expressing gag-pol (pMD.gag-pol) and env (VSV-G; pMD-G). Infections were carried out by diluting the viral supernatants with fresh medium (1:1), and spinning the resulting mix onto the target cells for $1 \mathrm{~h}$ at 2,000 r.p.m., $32^{\circ} \mathrm{C}$.

Cell lysis and co-immunoprecipitation assays. Cells were lysed in a buffer containing 1\% Igepal CA-630 detergent (Sigma), $50 \mathrm{mM}$ Tris $\mathrm{HCl} \mathrm{pH} \mathrm{7.5,} 150 \mathrm{mM}$ $\mathrm{NaCl}, 5 \mathrm{mM}$ EDTA and protease inhibitors (Sigma). After a 5 -min centrifugation step $\left(4^{\circ} \mathrm{C}\right)$, the resulting supernatants were evaluated for protein concentration (Bradford method, BioRad). For detection of endogenous LC3, t-ATG16L1 and $\mathrm{t}$-casp 3, cells were lysed by resuspending the cell pellet in $2 \mathrm{x}$ standard sample buffer containing $4 \%$ SDS but lacking $\beta$-mercaptoethanol and bromophenol blue, followed by extensive boiling. $\beta$-mercaptoethanol and bromophenol blue were added to the samples after measurement of protein concentrations. For co-immunoprecipitations with GST-fusion constructs, total cell lysates were diluted to a final detergent concentration of $0.2 \%$ and incubated $\left(4^{\circ} \mathrm{C}, 1-3 \mathrm{~h}\right.$, rotation) with agarose beads coupled to glutathione (GE Healthcare). Beads were then washed at least three times with immunoprecipitation buffer and resuspended in $2 \mathrm{x}$ SDS sample buffer.

Western blotting. Equal amounts of protein were resolved by SDS-polyacrylamide gel electrophoresis, transferred to a polyvinylidene-difluoride membrane (Millipore), and probed with specific antibodies against HA (monoclonal antibody (mAb), Babco MMS-101P, 1:1,000), AU1 (rabbit polyclonal, Babco PRB-130P, 1:1,000) or GFP (mAb, Babco MMS-118P, 1:1,000) tags, ATG16L1 (mAb, MBL M150-3, 1:1,000), tubulin (mAb, Sigma T4026, 1:40,000), GAPDH (mAb, Abcam ab8245, 1:10,000), LC3 (mAb, usually MBL M115-3, 1:1,000; MBL M186-3, 1:2,000, in Fig. 7 and Supplementary Fig. 8), human p62 (mAb, BD 610832, 1:1,000), mouse p62 (rabbit polyclonal, MBL PM045, 1:2,000), caspase 3 (rabbit polyclonal, Cell Signaling 9661, 1:1,000), GST (mAb, Santa Cruz sc-138, 1:1,000) or TMEM59 (rabbit polyclonal developed in-house) ${ }^{41}$. After incubation with appropriate secondary horseradish peroxidase-coupled antibodies (Jackson Immunoresearch), blots were developed by chemiluminescence (ECL system, Amersham). When re-probed, membranes were previously stripped for $15 \mathrm{~min}$ in a $7-\mathrm{M}$ guanidinium hydrochloride (Sigma) solution. The LC3-II/LC3-I signal intensity ratio was calculated after using the Image J software to analyse the relevant scanned films. Uncropped scanned images of the most relevant western blots are shown in Supplementary Fig. 10.

Pull-down assays. Isopropyl- $\beta$-D-thiogalactoside-induced bacteria (BL21) expressing the relevant GST fusion proteins (pGEX plasmid, GE Healthcare) were lysed by treatment with lysozyme $\left(100 \mu \mathrm{g} \mathrm{ml}^{-1}\right)$, freeze-thawing and sonication in a buffer containing $20 \mathrm{mM}$ Tris $\mathrm{HCl} \mathrm{pH} 8.0,500 \mathrm{mM} \mathrm{NaCl}, 1 \mathrm{mM}$ EDTA and $0.1 \%$ Triton X-100 (NET buffer). Lysates were cleared by centrifugation and incubated $\left(1 \mathrm{~h}, 4^{\circ} \mathrm{C}\right.$, rotation) with agarose beads coupled to glutathione (GE Healthcare). Loaded beads were washed extensively and used for ATG16L1 pull-down from induced crude bacterial lysates containing ATG16L1-T300 or -A300 proteins expressed from the pET plasmid (Novagen) in BL21(DE3) bacteria. Expression of these constructs in the soluble fraction was poor, but detectable. Beads were incubated with the amount of ATG16L1-containing lysate equivalent to $1 \mathrm{ml}$ of induced culture $\left(3 \mathrm{~h}, 4^{\circ} \mathrm{C}\right.$, rotation), washed and processed for western blotting.

Immunofluorescence studies and microscopy. Cells were seeded on poly-L-lysine coated coverslips, transfected, infected and/or treated the next day and fixed in $4 \%$ paraformaldehyde at the end of the experiment. Preparations were then permeabilized in a solution containing $0.5 \%$ Igepal CA-630 detergent, blocked in a solution containing 3\% BSA and stained with the relevant primary antibodies for $1 \mathrm{~h}$ at room temperature. Endogenous LC3 was stained using a rabbit anti-LC3 polyclonal antibody (MBL PM036, 1:600) except for experiments involving infection with $S$. aureus where a mouse monoclonal anti-LC3 antibody (MBL M152-3 (IgG1 low affinity for protein A) 1:50) was used. Fluorocrohome-coupled 
secondary antibodies were purchased from Jackson Immunoresearch (Cy3) or Molecular Probes (Alexa-488). No immunofluorescence signal was detected in Atg16l1-deficient MEFs infected with $S$. aureus, indicating that the antibody is unable to directly recognize the bacteria (through protein A, naturally expressed by this strain) under the experimental conditions used. For experiments involving CD16:7-263-281 transmembrane chimera, cells were transfected with the indicated constructs, split the next day onto poly-L-lysine coated coverslips and stimulated $36 \mathrm{~h}$ post transfection with an anti-CD16 antibody $\left(4 \mu \mathrm{g} \mathrm{ml}^{-1}, \mathrm{mAb}, \mathrm{NA} / \mathrm{LE}\right.$ formulation, BD 555403) followed by $10 \mu \mathrm{g} \mathrm{ml}^{-1}$ rabbit anti-mouse Fc $\gamma$ polyclonal serum (Jackson ImmunoResearch 315-005-008) to promote endocytosis. Aggregation was performed for $8 \mathrm{~h}$. To stain for endocytosed CD16:7 chimeras, unpermeabilized cells were quenched in vivo with unlabelled goat anti-mouse $\kappa$ chain antibody (Jackson ImmunoResearch 115-005-174, $60 \mathrm{\mu g} \mathrm{ml}^{-1}, 40 \mathrm{~min}, 4^{\circ} \mathrm{C}$, $0.1 \%$ azide) to remove the cell surface signal provided by the aggregating antibody. Cells were then fixed, permeabilized and stained with a goat anti-mouse $\kappa$ chain antibody conjugated to Cy3 (Jackson ImmunoResearch 115-165-174, 1:10,000). LC3 (both endogenous and transfected GFP-LC3) punctae quantification, TMEM59-GFP or CD16:7-263-281 vesicle counting and evaluation of GFP-expressing S. aureus in infected cells were conducted using a Zeiss Axiophot2 fluorescence microscope. Evaluation of endogenous LC3 punctae in cells transfected with TMEM59 was carried out by co-transfection with GFP and subsequent scoring of LC3 punctae (stained with a red fluorochrome; Cy3) only in GFP-positive cells. In cases where the relevant vesicles were accumulated in the perinuclear region (for example, TMEM59-GFP expressed in cells harbouring ATG16L1-T300) we inferred the number of vacuoles forming the irregular cluster by assessing the number of protruding lobuli. When indicated, samples were analysed under a Leica SP5 confocal microscope using the 488 (green), 561 (red) and/or $405 \mathrm{~nm}$ (blue) laser bands. Quantification of mRFP-LC3 intensity present on TMEM59-GFP vesicles was carried out over confocal images using the Intensity Quantification tool of the Leica Application Suite-Advance Fluorescence (LAS-AF) software.

Peptide microarrays. Expression and purification of the recombinant ligand from bacteria (GST-HA-ATG16L1-320-607) was performed using conventional techniques. Briefly, the construct was cloned in the pGEX bacterial expression vector and induced in mid-log BL21 bacterial cells using $10 \mathrm{nM}$ isopropyl- $\beta$-Dthiogalactoside at $25^{\circ} \mathrm{C}$ for $8 \mathrm{~h}$. Cells were lysed as described above (see 'Pull-down assays') and the clarified supernatants incubated with GSH-agarose beads for $3 \mathrm{~h}$ at $4{ }^{\circ} \mathrm{C}$. After extensive washing, the protein was eluted in $50 \mathrm{mM}$ reduced GSH and concentrated by centrifugation using a YM-30 Centricon filtering device (Amicon). A typical yield was $100-150 \mu \mathrm{g}$ of purified protein per litre of induced culture. The extended WDD form that includes residue 300 (ATG16L1-231-607) was unstable and poorly expressed in bacteria, as was full-length ATG16L1 (ref. 41). Expression and purification of the GST-HA-ATG16L1-231-607 construct from yeast cells was carried out using a modified version of the Kluyveromyces lactis Protein Expression Kit (New England Biolabs) where the protein was expressed without the secretion leader sequence included in the default vector (pKLAC2). Individual transformants were analysed for proper expression of the construct. The final cultures were grown to high density $(\mathrm{OD} 600=10)$ and mechanically lysed with glass beads (425-600 $\mu \mathrm{m}$, acid-washed, Sigma) and strong shaking provided by a FastPrep FP120 machine (BIO101 Savant) using 5 disruption cycles $(5 \mathrm{~s}$ shaking, 1 min off between cycles, $4^{\circ} \mathrm{C}$ ) at a 4.0 power setting. Lysates were clarified by centrifugation $\left(16,000 \mathrm{~g}, 10 \mathrm{~min}, 4^{\circ} \mathrm{C}\right)$ and the recombinant protein was recovered using GSH-agarose beads essentially as described above for expression in bacteria. To purify the recombinant ligands from mammalian cell lines, we used HEK-293T cells. Cells were transduced with retroviral constructs expressing the relevant ligands (GST-HA-ATG16L1-231-607, T300 or A300) followed by an IRESpuromycin element for selection ( $1 \mu \mathrm{g} \mathrm{ml}^{-1}$ of puromycin). Selected cultures were lysed in 1\% Igepal CA-630 lysis buffer. The resulting clarified lysates were diluted to a final Igepal CA-630 concentration of $0.2 \%$ and incubated with GSH-agarose beads for $4 \mathrm{~h}$ at $4{ }^{\circ} \mathrm{C}$. The recombinant protein was eluted from the washed matrix and concentrated as described above for the constructs expressed in bacteria. The approximate final yield was $1-2 \mu \mathrm{g}$ per $10-\mathrm{cm}$ plate $\left(\sim 10^{7}\right.$ cells $)$. All samples were run in protein gels and stained with Coomassie blue to evaluate integrity and approximate protein quantity. A more accurate quantification was obtained using the DC Protein Assay kit (BioRad).

Peptide microarrays were ordered from the company JPT (Germany). All peptides were printed in triplicates in three different sub-arrays (nine spots per peptide in each microarray). Peptides were immobilized through the N-terminus using a long spacer (Tds), and some of them also included a biotin group between the spacer and the peptide to evaluate the amount of peptide printed in each spot. Slides were first blocked in a solution containing PBS/0.05\% Tween-20 (PBS-T) supplemented with $5 \%$ BSA for $2 \mathrm{~h}$ at room temperature, and then washed in PBS$\mathrm{T}$ with gentle shaking. Ligand incubation was performed in blocking solution containing the desired concentration of the recombinant protein $(400 \mathrm{nM}$ to $1 \mu \mathrm{M}$, as indicated in the relevant figure legends) for $2 \mathrm{~h}$ at room temperature, then overnight at $4^{\circ} \mathrm{C}$. After washing, slides were incubated with a mixture of anti-HA (mAb; Babco MMS-101P) and anti-GST (rabbit polyclonal; Cell Signaling 2622) antibodies $\left(10 \mu \mathrm{g} \mathrm{ml}^{-1}\right.$ each, diluted in blocking buffer) for $2 \mathrm{~h}$ at room temperature. Arrays were then incubated with a solution containing a mixture of
Cy5-labelled goat anti-mouse and anti-rabbit (Jackson ImmunoResearch) antibodies $\left(1.5 \mu \mathrm{g} \mathrm{ml}^{-1}\right.$ each in blocking buffer, $1 \mathrm{~h}$ at room temperature) followed by repeated washing in PBS-T. The anti-biotin antibody was purchased from Jackson ImmunoResearch (mAb, 200-002-211) and used at $10 \mu \mathrm{g} \mathrm{ml}^{-1}$ to directly probe the arrays, followed by incubation with a goat anti-mouse secondary antibody coupled to Cy5. A final wash with MilliQ distilled water allowed removal of salt residues. Slides were scanned using a GenePix 4000B slide scanner (Molecular Devices) and the resulting images analysed with the GenePix Pro software. The background signal provided by an irrelevant peptide present in the same array (FLAG) was subtracted from the relevant binding values before further processing the data for each peptide. Such background noise was typically $40-50$ times lower than the specific signal.

Bacterial strains and infection assays. Cells were infected with a GFP-expressing transformant of the S. aureus strain RN6390 (ref. 64) kindly provided by $\mathrm{Dr}$ A. Cheung. The indicated cell lines were incubated with the bacteria for $45 \mathrm{~min}$, washed and treated with $100 \mathrm{\mu g} \mathrm{ml}^{-1}$ gentamicin for $1 \mathrm{~h}$ to inhibit extracellular proliferation of $S$. aureus. This was considered the $2 \mathrm{~h}$ time point at which cells were processed for further analysis. Determination of colony-forming units recovered from infected cells was done by lysing the infected cells in $0.1 \%$ Triton $\mathrm{X}-100$ for $3 \mathrm{~min}$ and plating serial dilutions of the lysates in brain heart infusion (BHI)-agar plates for subsequent colony quantification.

siRNA assays. MEFs were transfected with pre-designed pools of four RNA duplexes against mouse Tmem59 (On-TargetPlus, Dharmacon L-059473-01) using the transfection reagent DharmaFECT1 (Dharmacon) following the instructions provided by the manufacturers. Control siRNAs were a mix of duplexes having no perfect match with any mouse gene (On-TargetPlus Non-targeting pool, Dharmacon D-001810-10). Since Tmem59 expression levels are normally below the detection threshold of our antibody ${ }^{41}$, the degree of protein depletion caused by siRNA treatment was assessed after induction of Tmem59 expression by treatment with bafilomycin $48 \mathrm{~h}$ after transfection with the relevant siRNAs.

Data availability. The data that support the findings of this study are available from the corresponding author on request.

\section{References}

1. Sartor, R. B. Mechanisms of disease: pathogenesis of Crohn's disease and ulcerative colitis. Nat. Clin. Pract. Gastroenterol. Hepatol. 3, 390-407 (2006).

2. Abraham, C. \& Cho, J. H. Inflammatory bowel disease. N. Engl. J. Med. 361, 2066-2078 (2009).

3. Khor, B., Gardet, A. \& Xavier, R. J. Genetics and pathogenesis of inflammatory bowel disease. Nature 474, 307-317 (2011).

4. Stappenbeck, T. S. et al. Crohn disease: a current perspective on genetics, autophagy and immunity. Autophagy 7, 355-374 (2011).

5. Podolsky, D. K. Inflammatory bowel disease. N. Engl. J. Med. 347, 417-429 (2002).

6. Rioux, J. D. et al. Genome-wide association study identifies new susceptibility loci for Crohn disease and implicates autophagy in disease pathogenesis. Nat. Genet. 39, 596-604 (2007).

7. Hampe, J. et al. A genome-wide association scan of nonsynonymous SNPs identifies a susceptibility variant for Crohn disease in ATG16L1. Nat. Genet. 39, 207-211 (2007).

8. Hugot, J. P. et al. Association of NOD2 leucine-rich repeat variants with susceptibility to Crohn's disease. Nature 411, 599-603 (2001).

9. Ogura, Y. et al. A frameshift mutation in NOD2 associated with susceptibility to Crohn's disease. Nature 411, 603-606 (2001).

10. Duerr, R. H. et al. A genome-wide association study identifies IL23R as an inflammatory bowel disease gene. Science 314, 1461-1463 (2006).

11. Lapaquette, P., Brest, P., Hofman, P. \& Darfeuille-Michaud, A. Etiology of Crohn's disease: many roads lead to autophagy. J. Mol. Med. (Berl.) 90, 987-996 (2012).

12. Mizushima, N. \& Komatsu, M. Autophagy: renovation of cells and tissues. Cell 147, 728-741 (2011)

13. Kroemer, G., Marino, G. \& Levine, B. Autophagy and the integrated stress response. Mol. Cell 40, 280-293 (2010).

14. Feng, Y., He, D., Yao, Z. \& Klionsky, D. J. The machinery of macroautophagy. Cell Res. 24, 24-41 (2014).

15. Sumpter, Jr R. \& Levine, B. Autophagy and innate immunity: triggering, targeting and tuning. Semin. Cell Dev. Biol. 21, 699-711 (2010).

16. Noda, T. \& Yoshimori, T. Molecular basis of canonical and bactericidal autophagy. Int. Immunol. 21, 1199-1204 (2009).

17. Pimentel-Muiños, F. X. \& Boada-Romero, E. Selective autophagy against membranous compartments: canonical and unconventional purposes and mechanisms. Autophagy 10, 397-407 (2014).

18. Levine, B., Mizushima, N. \& Virgin, H. W. Autophagy in immunity and inflammation. Nature 469, 323-335 (2011). 
19. Gomes, L. C. \& Dikic, I. Autophagy in antimicrobial immunity. Mol. Cell 54, 224-233 (2014).

20. Deretic, V. \& Levine, B. Autophagy, immunity, and microbial adaptations. Cell Host Microbe 5, 527-549 (2009).

21. Caruso, R., Warner, N., Inohara, N. \& Nunez, G. NOD1 and NOD2: signaling, host defense, and inflammatory disease. Immunity 41, 898-908 (2014).

22. Plantinga, T. S., Joosten, L. A., van der Meer, J. W. \& Netea, M. G. Modulation of inflammation by autophagy: consequences for Crohn's disease. Curr. Opin. Pharmacol. 12, 497-502 (2012).

23. Nguyen, H. T., Lapaquette, P., Bringer, M. A. \& Darfeuille-Michaud, A. Autophagy and Crohn's disease. J Innate Immun. 5, 434-443 (2013).

24. Fujita, N. et al. The Atg16L complex specifies the site of LC3 lipidation for membrane biogenesis in autophagy. Mol. Biol. Cell 19, 2092-2100 (2008).

25. He, C. \& Klionsky, D. J. Regulation mechanisms and signaling pathways of autophagy. Annu. Rev. Genet. 43, 67-93 (2009).

26. Hanada, T. et al. The Atg12-Atg5 conjugate has a novel E3-like activity for protein lipidation in autophagy. J. Biol. Chem. 282, 37298-37302 (2007).

27. Mizushima, N. et al. Mouse Apg16L, a novel WD-repeat protein, targets to the autophagic isolation membrane with the Apg12-Apg5 conjugate. J. Cell Sci. 116, 1679-1688 (2003).

28. Nishimura, T. et al. FIP200 regulates targeting of Atg16L1 to the isolation membrane. EMBO. Rep. 14, 284-291 (2013).

29. Gammoh, N., Florey, O., Overholtzer, M. \& Jiang, X. Interaction between FIP200 and ATG16L1 distinguishes ULK1 complex-dependent and independent autophagy. Nat. Struct. Mol. Biol. 20, 144-149 (2013).

30. Fujita, N. et al. Differential involvement of Atg16L1 in Crohn disease and canonical autophagy: analysis of the organization of the Atg16L1 complex in fibroblasts. J. Biol. Chem. 284, 32602-32609 (2009).

31. Choi, J. et al. The parasitophorous vacuole membrane of Toxoplasma gondii is targeted for disruption by ubiquitin-like conjugation systems of autophagy. Immunity 40, 924-935 (2014).

32. Dooley, H. C. et al. WIPI2 links LC3 conjugation with PI3P, autophagosome formation, and pathogen clearance by recruiting Atg12-5-16L1. Mol. Cell 55, 238-252 (2014)

33. Itoh, T. et al. Golgi-resident small GTPase Rab33B interacts with Atg16L and modulates autophagosome formation. Mol. Biol. Cell 19, 2916-2925 (2008).

34. Sorbara, M. T. et al. The protein ATG16L1 suppresses inflammatory cytokines induced by the intracellular sensors Nod1 and Nod2 in an autophagy-independent manner. Immunity 39, 858-873 (2013).

35. Saitoh, T. et al. Loss of the autophagy protein Atg16L1 enhances endotoxin-induced IL-1beta production. Nature 456, 264-268 (2008).

36. Conway, K. L. et al. Atg16l1 is required for autophagy in intestinal epithelial cells and protection of mice from Salmonella infection. Gastroenterology 145 1347-1357 (2013).

37. Cadwell, K. et al. Virus-plus-susceptibility gene interaction determines Crohn's disease gene Atg16L1 phenotypes in intestine. Cell 141, 1135-1145 (2010).

38. Cadwell, K. et al. A key role for autophagy and the autophagy gene Atg1611 in mouse and human intestinal Paneth cells. Nature 456, 259-263 (2008).

39. Kuballa, P., Huett, A., Rioux, J. D., Daly, M. J. \& Xavier, R. J. Impaired autophagy of an intracellular pathogen induced by a Crohn's disease associated ATG16L1 variant. PLoS ONE 3, e3391 (2008).

40. Travassos, L. H. et al. Nod1 and Nod2 direct autophagy by recruiting ATG16L1 to the plasma membrane at the site of bacterial entry. Nat. Immunol. 11, 55-62 (2010).

41. Boada-Romero, E. et al. TMEM59 defines a novel ATG16L1-binding motif that promotes local activation of LC3. EMBO J. 32, 566-582 (2013).

42. Ullrich, S. et al. The novel membrane protein TMEM59 modulates complex glycosylation, cell surface expression, and secretion of the amyloid precursor protein. J. Biol. Chem. 285, 20664-20674 (2010).

43. Fujita, N. et al. Recruitment of the autophagic machinery to endosomes during infection is mediated by ubiquitin. J. Cell Biol. 203, 115-128 (2013).

44. Pashkova, N. et al. WD40 repeat propellers define a ubiquitin-binding domain that regulates turnover of F box proteins. Mol. Cell 40, 433-443 (2010).

45. Messer, J. S. et al. The Crohn's disease: associated ATG16L1 variant and Salmonella invasion. BMJ Open 3, e002790 (2013).

46. Lassen, K. G. et al. Atg16L1 T300A variant decreases selective autophagy resulting in altered cytokine signaling and decreased antibacterial defense. Proc. Natl Acad. Sci. USA 111, 7741-7746 (2014)

47. Murthy, A. et al. A Crohn's disease variant in Atg16l1 enhances its degradation by caspase 3. Nature 506, 456-462 (2014).

48. Homer, C. R., Richmond, A. L., Rebert, N. A., Achkar, J. P. \& McDonald, C. ATG16L1 and NOD2 interact in an autophagy-dependent antibacterial pathway implicated in Crohn's disease pathogenesis. Gastroenterology 139, 1630-1641 1641 e1-2 (2010).

49. Cooney, R. et al. NOD2 stimulation induces autophagy in dendritic cells influencing bacterial handling and antigen presentation. Nat. Med. 16, 90-97 (2010).

50. Klionsky, D. J. \& Schulman, B. A. Dynamic regulation of macroautophagy by distinctive ubiquitin-like proteins. Nat. Struct. Mol. Biol. 21, 336-345 (2014).
51. Plantinga, T. S. et al. Crohn's disease-associated ATG16L1 polymorphism modulates pro-inflammatory cytokine responses selectively upon activation of NOD2. Gut 60, 1229-1235 (2011).

52. Dadgostar, H., Doyle, S. E., Shahangian, A., Garcia, D. E. \& Cheng, G. T3JAM, a novel protein that specifically interacts with TRAF3 and promotes the activation of JNK(1). FEBS Lett. 553, 403-407 (2003).

53. Roth, W., Stenner-Liewen, F., Pawlowski, K., Godzik, A. \& Reed, J. C. Identification and characterization of DEDD2, a death effector domaincontaining protein. J. Biol. Chem. 277, 7501-7508 (2002).

54. Roy, P. K., Rashid, F., Bragg, J. \& Ibdah, J. A. Role of the JNK signal transduction pathway in inflammatory bowel disease. World J. Gastroenterol. 14, 200-202 (2008).

55. Hausmann, M. How bacteria-induced apoptosis of intestinal epithelial cells contributes to mucosal inflammation. Int. J. Inflam. 2010, 574568 (2010).

56. Maurer, K. et al. Autophagy mediates tolerance to Staphylococcus aureus alpha-toxin. Cell Host Microbe 17, 429-440 (2015).

57. Schnaith, A. et al. Staphylococcus aureus subvert autophagy for induction of caspase-independent host cell death. J. Biol. Chem. 282, 2695-2706 (2007).

58. Hubbard-Lucey, V. M. et al. Autophagy gene Atg16L1 prevents lethal T cell alloreactivity mediated by dendritic cells. Immunity 41, 579-591 (2014).

59. Grimm, W. A. et al. The Thr300Ala variant in ATG16L1 is associated with improved survival in human colorectal cancer and enhanced production of type I interferon. Gut 65, 456-464 (2016).

60. Burada, F. et al. ATG16L1 T300A polymorphism is correlated with gastric cancer susceptibility. Pathol. Oncol. Res. 22, 317-322 (2016).

61. Usategui-Martin, R. et al. Polymorphisms in autophagy genes are associated with paget disease of bone. PLoS ONE 10, e0128984 (2015).

62. Stennicke, H. R., Renatus, M., Meldal, M. \& Salvesen, G. S. Internally quenched fluorescent peptide substrates disclose the subsite preferences of human caspases 1, 3, 6, 7 and 8. Biochem. J. 350(Pt 2): 563-568 (2000).

63. Klee, M., Pallauf, K., Alcalá, S., Fleischer, A. \& Pimentel-Muiños, F. X. Mitochondrial apoptosis induced by $\mathrm{BH} 3$-only molecules in the exclusive presence of endoplasmic reticular Bak. EMBO J. 28, 1757-1768 (2009).

64. Kahl, B. C. et al. Staphylococcus aureus RN6390 replicates and induces apoptosis in a pulmonary epithelial cell line. Infect. Immun. 68, 5385-5392 (2000).

\section{Acknowledgements}

We thank members of the CIC for support. This work was funded by grants from the Ministerio de Ciencia e Innovación (Ref. SAF2011-23714) and the Ministerio de Economía y Competitividad (Ref. SAF2014-53320-R) of the Spanish Government, the Broad Medical Research Program at Crohn's and Colitis Foundation of America (CCFA Ref. IBD-0369), the Junta de Castilla y León local government (Department of Education (Refs. CSI001A10-2, FIC016U14) and Department of Health (Ref. SAN11-FXP)) and the Fundación Solórzano (Refs. FS/1-2009 and FS/18-2014). R.J.X. is funded by the NIH (grants R01DK097485, P30DK043351 and U19AI109725) and the Helmsley Trust. Additional funding comes from the FEDER programme of the European Union. E.B.R and I.S.G. are graduate students funded by predoctoral fellowships from the FPU programme (Ministerio de Educación, MEC, Spanish Government) and the Fundación Moraza, respectively. F.X.P. holds a tenured position at the CSIC.

\section{Author contributions}

E.B.R. and I.S.G. performed the experiments. M.P.S. carried out experimental work during the revision process. D.L.B. and R.J.X. provided cellular reagents as well as critical reading and editing of the manuscript. F.X.P. supervised the research, designed the experiments and wrote the manuscript.

\section{Additional information}

Supplementary Information accompanies this paper at http://www.nature.com/ naturecommunications

Competing financial interests: The authors declare no competing financial interests.

Reprints and permission information is available online at http://npg.nature.com/ reprintsandpermissions/

How to cite this article: Boada-Romero, E. et al. The T300A Crohn's disease risk polymorphism impairs function of the WD40 domain of ATG16L1. Nat. Commun. 7:11821 doi: 10.1038/ncomms11821 (2016).

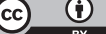

This work is licensed under a Creative Commons Attribution 4.0 International License. The images or other third party material in this article are included in the article's Creative Commons license, unless indicated otherwise in the credit line; if the material is not included under the Creative Commons license, users will need to obtain permission from the license holder to reproduce the material. To view a copy of this license, visit http://creativecommons.org/licenses/by/4.0/ 